Article

\title{
Fractional Order of Evolution Inclusion Coupled with a Time and State Dependent Maximal Monotone Operator
}

\author{
Charles Castaing ${ }^{1, *}$, Christiane Godet-Thobie ${ }^{2}$ (D) and Le Xuan Truong ${ }^{3}$ \\ 1 Département de Mathématiques, Université Montpellier II, Case Courrier 051, \\ 34095 Montpellier CEDEX 5, France \\ 2 Laboratoire de Mathématiques de Bretagne Atlantique, Université de Bretagne Occidentale, \\ CNRS UMR 6205, 6, Avenue Victor Le Gorgeu, CS 9387, F-29238 Brest CEDEX 3, France; \\ christiane.godet-thobie@univ-brest.fr \\ 3 Department of Mathematics and Statistics, University of Economics Ho Chi Minh City, \\ Ho Chi Minh City 700000, Vietnam; lxuantruong@gmail.com \\ * Correspondence: christiane.godet-thobie@univ-brest.fr
}

Received: 5 July 2020; Accepted: 11 August 2020; Published: 20 August 2020

\begin{abstract}
This paper is devoted to the study of evolution problems involving fractional flow and time and state dependent maximal monotone operator which is absolutely continuous in variation with respect to the Vladimirov's pseudo distance. In a first part, we solve a second order problem and give an application to sweeping process. In a second part, we study a class of fractional order problem driven by a time and state dependent maximal monotone operator with a Lipschitz perturbation in a separable Hilbert space. In the last part, we establish a Filippov theorem and a relaxation variant for fractional differential inclusion in a separable Banach space. In every part, some variants and applications are presented.
\end{abstract}

Keywords: fractional differential inclusion; maximal monotone operator; Riemann-Liouville integral; absolutely continuous in variation; Vladimirov pseudo-distance

MSC: 34H05; 34K35; 47H10; 28A25; 28B20; 28C20

\section{Introduction}

In recent decades, fractional equations and inclusions have proven to be interesting tools in the modeling of many physical or economic phenomena. In addition, there has been a significant development in fractional differential theory and applications in recent years [1-7]. In the case of the sole inclusion, $D^{\alpha} u(t) \in F(t, u(t))$, one can find an important piece of literature. For examples, in following papers, study is made with different boundary conditions [8-12], with use of the non-compactness measure $[13,14]$, with use of contraction principle in the space of selections of the set valued map instead in the space of solutions [15], with compactness conditions [16] or inclusions with infinite delay [17]. To the best of our acknowledge, a very few study is available in the fractional order differential inclusion coupled with a time and state dependent maximal monotone operator ([18] with subdifferential operators).

The main objective of the present work is to develop the existence theory for a coupled system of evolution inclusion driven by fractional differential equation and time and state dependent maximal monotone operators. The developments of the article are as follows. 
At first, we investigate a second order problem governed a time and state dependent maximal monotone operator with Lipschitz perturbation in a separable Hilbert space $E$ (The second order is in the state variable $x$ ).

$$
\text { (1.1) }\left\{\begin{array}{l}
x(t)=x_{0}+\int_{0}^{t} u(s) d s, t \in[0, T] \\
u(t) \in D\left(A_{t, x(t)}\right), t \in[0, T] \\
-\dot{u}(t) \in A_{t, x(t)} u(t)+f(t, x(t), u(t)) \quad \text { a.e. }
\end{array}\right.
$$

Secondly, we investigate a class of fractional order problem driven by a time and state dependent maximal monotone operator with Lipschitz perturbation in $E$ of the form

$$
(1.2)\left\{\begin{array}{l}
D^{\alpha} h(t)+\lambda D^{\alpha-1} h(t)=u(t), t \in[0,1] \\
\left.I_{0^{+}}^{\beta} h(t)\right|_{t=0}:=\lim _{t \rightarrow 0} \int_{0}^{t} \frac{(t-s)^{\beta-1}}{\Gamma(\beta)} h(s) d s=0, \quad h(1)=I_{0^{+}}^{\gamma} h(1)=\int_{0}^{1} \frac{(1-s)^{\gamma-1}}{\Gamma(\gamma)} h(s) d s \\
-\dot{u}(t) \in A_{t, h(t)} u(t)+f(t, h(t), u(t)) \quad \text { a.e. }
\end{array}\right.
$$

where $\alpha \in] 1,2], \beta \in[0,2-\alpha], \lambda \geq 0, \gamma>0$ are given constants, $D^{\alpha}$ is the standard Riemann-Liouville fractional derivative, $\Gamma$ is the gamma function, $(t, x) \rightarrow A_{(t, x)}: D\left(A_{(t, x)}\right) \rightarrow 2^{E}$ is a maximal monotone operator with domain $D\left(A_{(t, x)}\right)$ and $f:[0,1] \times E \times E \rightarrow E$ is a single valued Lipschitz perturbation w.r.t $y \in E$.

Thirdly, we finish the paper with a Fillipov theorem and relaxation theorem for fractional differential inclusion in a separable Banach space $E$

$$
\left(\mathcal{P}_{F}\right)\left\{\begin{array}{l}
D^{\alpha} u(t)+\lambda D^{\alpha-1} u(t) \in F(t, u(t)), \text { a.e. } t \in[0,1] \\
\left.I_{0^{+}}^{\beta} u(t)\right|_{t=0}=0, \quad u(1)=I_{0^{+}}^{\gamma} u(1)
\end{array}\right.
$$

and

$$
\left(\mathcal{P}_{\overline{c o} F}\right)\left\{\begin{array}{l}
D^{\alpha} u(t)+\lambda D^{\alpha-1} u(t) \in \overline{c o} F(t, u(t)), \text { a.e. } t \in[0,1] \\
\left.I_{0^{+}}^{\beta} u(t)\right|_{t=0}=0, \quad u(1)=I_{0^{+}}^{\gamma} u(1)
\end{array}\right.
$$

where $F$ is closed valued $\mathcal{L}(I) \times \mathcal{B}(E)$-measurable and Lipschitz w.r.t $x \in E$.

Within the framework of studies concerning coupled systems of evolution inclusion driven by fractional differential equation and time and state dependent maximal monotone operator, our results are fairly general and new and give further insight into the characteristics of both evolution inclusion and fractional order boundary value problems.

\section{Notations and Preliminaries}

In the whole paper, $I:=[0, T](T>0)$ is an interval of $\mathbb{R}$ and $E$ is a separable Hilbert space with the scalar product $\langle\cdot, \cdot\rangle$ and the associated norm $\|\cdot\| . \bar{B}_{E}$ denotes the unit closed ball of $E$ and $r \bar{B}_{E}$ its closed ball of center 0 and radius $r>0$. We denote by $\mathcal{L}(I)$ the sigma algebra on $I, \lambda:=d t$ the Lebesgue measure and $\mathcal{B}(E)$ the Borel sigma algebra on $E$. If $\mu$ is a positive measure on $I$, we will denote by $L^{p}(I, E, \mu) p \in[1,+\infty[$, (resp. $p=+\infty$ ), the Banach space of classes of measurable functions $u: I \rightarrow E$ such that $t \mapsto\|u(t)\|^{p}$ is $\mu$-integrable (resp. $u$ is $\mu$-essentially bounded), equipped with its classical norm $\|\cdot\|_{p}$ (resp. $\|\cdot\|_{\infty}$ ). We denote by $\mathcal{C}(I, E)$ the Banach space of all continuous mappings $u: I \rightarrow E$, endowed with the sup norm.

The excess between closed subsets $C_{1}$ and $C_{2}$ of $E$ is defined by $e\left(C_{1}, C_{2}\right):=\sup _{x \in C_{1}} d\left(x, C_{2}\right)$, and the Hausdorff distance between them is given by

$$
d_{H}\left(C_{1}, C_{2}\right):=\max \left\{e\left(C_{1}, C_{2}\right), e\left(C_{2}, C_{1}\right)\right\} .
$$

The support function of $S \subset E$ is defined by: $\delta^{*}(a, S):=\sup _{x \in S}\langle a, x\rangle, \forall a \in E$.

If $X$ is a Banach space and $X^{*}$ its topological dual, we denote by $\sigma\left(X, X^{*}\right)$ the weak topology on $X$, and by $\sigma\left(X^{*}, X\right)$ the weak* topology on $X^{*}$. 
Let $A: E \rightrightarrows E$ be a set-valued map. We denote by $D(A), R(A)$ and $G r(A)$ its domain, range and graph. We say that $A$ is monotone, if $\left\langle y_{1}-y_{2}, x_{1}-x_{2}\right\rangle \geq 0$ whenever $x_{i} \in D(A)$, and $y_{i} \in A\left(x_{i}\right)$, $i=1,2$. In addition, we say that $A$ is a maximal monotone operator of $E$, if its graph could not be contained properly in the graph of any other monotone operator. By Minty's Theorem, $A$ is maximal monotone iff $R\left(I_{E}+A\right)=E$.

If $A$ is a maximal monotone operator of $E$, then, for every $x \in D(A), A(x)$ is nonempty closed and convex. We denote the projection of the origin on the set $A(x)$ by $A^{0}(x)$.

Let $\lambda>0$; then, the resolvent and the Yosida approximation of $A$ are the well-known operators defined respectively by $J_{\lambda}^{A}=\left(I_{E}+\lambda A\right)^{-1}$ and $A_{\lambda}=\frac{1}{\lambda}\left(I_{E}-J_{\lambda}^{A}\right)$. These operators are single-valued and defined on all of $E$, and we have $J_{\lambda}^{A}(x) \in D(A)$, for all $x \in E$. For more details about the theory of maximal monotone operators, we refer the reader to $[5,19,20]$.

Let $A: D(A) \subset E \rightarrow 2^{E}$ and $B: D(B) \subset E \rightarrow 2^{E}$ be two maximal monotone operators, then we denote by $\operatorname{dis}(A, B)$ the pseudo-distance between $A$ and $B$ defined by

$$
\operatorname{dis}(A, B)=\sup \left\{\frac{\left\langle y-y^{\prime}, x^{\prime}-x\right\rangle}{1+\|y\|+\left\|y^{\prime}\right\|}: x \in D(A), y \in A x, x^{\prime} \in D(B), y^{\prime} \in B x^{\prime}\right\} \text {. }
$$

This pseudo-distance due to Vladimiro [21] is particularly well suited to the study of operators (see its use in [22]) and also, in the sweeping process, for its links with the Hausdorff distance in convex analysis. Indeed, if $N_{C(t, x)}$ is the normal cone of the closed convex set $C(t, x)$, we have

$$
\operatorname{dis}\left(N_{C(t, x)}, N_{C(s, y)}\right)=d_{H}(C(t, x), C(s, y)) \text {. }
$$

This property will be used in this paper.

For the proof of our main theorems, we will need some elementary lemmas taken from reference [23].

Lemma 1. Let $A$ be a maximal monotone operator of $E$. If $x \in \overline{D(A))}$ and $y \in E$ are such that

$$
\left\langle A^{0}(z)-y, z-x\right\rangle \geq 0 \forall z \in D(A)
$$

then $x \in D(A)$ and $y \in A(x)$.

Lemma 2. Let $A_{n}(n \in \mathbb{N})$, A be maximal monotone operators of $E$ such that dis $\left(A_{n}, A\right) \rightarrow 0$. Suppose also that $x_{n} \in D\left(A_{n}\right)$ with $x_{n} \rightarrow x$ and $y_{n} \in A_{n}\left(x_{n}\right)$ with $y_{n} \rightarrow y$ weakly for some $x, y \in E$. Then, $x \in D(A)$ and $y \in A(x)$.

Lemma 3. Let $A, B$ be maximal monotone operators of $E$. Then,

(1) for $\lambda>0$ and $x \in D(A)$

$$
\left\|x-J_{\lambda}^{B}(x)\right\| \leq \lambda\left\|A^{0}(x)\right\|+\operatorname{dis}(A, B)+\sqrt{\lambda\left(1+\left\|A^{0}(x)\right\|\right) \operatorname{dis}(A, B)} .
$$

(2) For $\lambda>0$ and $x, x^{\prime} \in E$

$$
\left\|J_{\lambda}^{A}(x)-J_{\lambda}^{A}\left(x^{\prime}\right)\right\| \leq\left\|x-x^{\prime}\right\|
$$

Lemma 4. Let $A_{n}(n \in \mathbb{N})$, A be maximal monotone operators of $E$ such that $\operatorname{dis}\left(A_{n}, A\right) \rightarrow 0$ and $\left\|A_{n}^{0}(x)\right\| \leq$ $c(1+\|x\|)$ for some $c>0$, all $n \in \mathbb{N}$ and $x \in D\left(A_{n}\right)$. Then, for every $z \in D(A)$, there exists a sequence $\left(\zeta_{n}\right)$ such that

$$
\zeta_{n} \in D\left(A_{n}\right), \quad \zeta_{n} \rightarrow z \text { and } A_{n}^{0}\left(\zeta_{n}\right) \rightarrow A^{0}(z)
$$




\section{On Second Order Problem Driven by a Time and State Dependent Maximal Operator}

Let $I=[0, T]$ and let $E$ be a separable Hilbert space. In this part, we are interested in solving the problem (1.1).

Lemma 5. Let $(t, x) \rightarrow A_{(t, x)}: D\left(A_{(t, x)}\right) \rightarrow 2^{E}$ a maximal monotone operator satisfying:

$\left(H_{1}\right)\left\|A_{(t, x)}^{0} y\right\| \leq c(1+\|x\|+\|y\|)$ for all $(t, x, y) \in I \times E \times D\left(A_{(t, x)}\right)$, for some positive constant $c$,

$\left(H_{2}\right) \operatorname{dis}\left(A_{(t, x)}, A_{(\tau, y)}\right) \leq a(t)-a(\tau)+r\|x-y\|$, for all $0 \leq \tau \leq t \leq T$ and for all $(x, y) \in E \times E$, where $r$ is a positive number, $a: I \rightarrow\left[0,+\infty\left[\right.\right.$ is nondecreasing absolutely continuous on $I$ with $\dot{a} \in L^{2}$, shortly $a \in W^{1,2}(I)$.

Then, the following hold:

Fact $\mathcal{I}$ : For any absolutely continuous $x \in W_{E}^{1,2}(I)$ and for any $u_{0} \in D\left(A_{(0, x(0))}\right)$, the problem

$$
\left\{\begin{array}{l}
-\dot{u}(t) \in A_{(t, x(t))} u(t), \text { a.e. } t \in I \\
u(t) \in D\left(A_{(t, x(t))}\right), \forall t \in I \\
u(0)=u_{0} \in D\left(A_{(0, x(0))}\right)
\end{array}\right.
$$

has a unique absolutely continuous solution with $\|\dot{u}(t)\| \leq K(1+\dot{\beta}(t))$ where $\beta(t)=$ $\left.\int_{0}^{t}[\dot{a}(s)+r \| \dot{x}(s)) \|\right] d s, \forall t \in I$ and $K$ is a positive constant depending on $\left\|u_{0}\right\|, c, T, x$ and $\beta$.

Fact $\mathcal{J}$ : Assume that

$\left(H_{3}\right)(t, x, y) \rightarrow J_{\lambda}^{A_{(t, x)}}(y)$ is $\mathcal{L}(I) \otimes \mathcal{B}(E) \otimes \mathcal{B}(E)$-measurable.

Then, the composition operator $\mathcal{A}_{x}: D\left(\mathcal{A}_{x}\right) \subset L^{2}(I, E, d t) \rightarrow 2^{L^{2}(I, E, d t)}$ defined by

$$
\mathcal{A}_{x} u=\left\{v \in L^{2}(I, E, d t): v(t) \in A_{(t, x(t))} u(t) \text { a.e. } t \in I\right\}
$$

for each $u \in D\left(\mathcal{A}_{x}\right)$ where

$D\left(\mathcal{A}_{x}\right):=\left\{u \in L^{2}(I, E, d t): u(t) \in D\left(A_{(t, x(t))}\right)\right.$ a.e. $t \in I$, for which $\exists y \in L^{2}(I, E, d t): y(t) \in$ $A_{(t, x(t))} u(t)$, a.e. $\left.t \in I\right\}$

is maximal monotone. Consequently, the graph of $\mathcal{A}_{x}: D\left(\mathcal{A}_{x}\right) \subset L^{2}(I, E, d t) \rightarrow 2^{L^{2}(I, E, d t)}$ is strongly-weakly sequentially closed in $L^{2}(I, E, d t) \times L^{2}(I, E, d t)$.

Proof. Fact $\mathcal{I}$. The mapping $B_{t}=A_{(t, x(t))}$ is a time dependent absolutely continuous in variation maximal monotone operator: For all $0 \leq \tau \leq t \leq T$, we have by $\left(H_{2}\right)$

$$
\left\{\begin{array}{l}
\operatorname{dis}\left(B_{t}, B_{\tau}\right)=\operatorname{dis}\left(A_{(t, x(t))}, A_{(\tau, x(\tau))}\right) \\
\leq|a(t)-a(\tau)|+r\|x(t)-x(\tau)\| \\
\leq \int_{\tau}^{t} \dot{a}(s) d s+r \int_{\tau}^{t}\|\dot{x}(s)\| d s \\
=\beta(t)-\beta(\tau)
\end{array}\right.
$$

where $\beta(t)=\int_{0}^{t}[\dot{a}(s)+r\|\dot{x}(s)\|] d s, \forall t \in I$. Furthermore, by $\left(H_{1}\right)$, we have

$$
\left\{\begin{array}{l}
\left\|B_{t}^{0} y\right\|=\left\|A_{(t, x(t))}^{0} y\right\| \leq c(1+\|x(t)\|+\|y\|) \\
\leq c_{1}(1+\|y\|)
\end{array}\right.
$$


for all $y \in D\left(A_{(t, x(t))}\right)$, where $c_{1}$ is a positive generic constant. Consequently, by [22] (Theorem 3.5), for every $u_{0} \in D\left(B_{0}\right)$, a unique absolutely continuous mapping $u: I \rightarrow E$ exists satisfying

$$
\left\{\begin{array}{l}
-\dot{u}(t) \in B_{t} u(t)=A_{(t, x(t))} u(t), \text { a.e. } t \in I \\
u(t) \in D\left(B_{t}\right)=D\left(A_{(t, x(t))}\right), \forall t \in I \\
u(0)=u_{0} \in D\left(B_{0}\right)=D\left(A_{(0, x(0))}\right)
\end{array}\right.
$$

with $\|\dot{u}(t)\| \leq K(1+\dot{\beta}(t))$, where $\left.\beta(t)=\int_{0}^{t}[\dot{a}(s)+r \| \dot{x}(s)) \|\right] d s, \forall t \in I$ and $K$ is a positive constant depending on $\left\|u_{0}\right\|, c, T, \beta$.

Fact $\mathcal{J}$. Taking account $\mathcal{J}$, it is clear that $D\left(\mathcal{A}_{x}\right)$ is nonempty and $\mathcal{A}_{x}$ is well defined. It is easy to see that $\mathcal{A}_{x}$ is monotone. Let us prove that $\mathcal{A}_{x}$ is maximal monotone. We have to check that $R\left(I_{L^{2}(I, E, d t)}+\lambda \mathcal{A}_{x}\right)=L^{2}(I, E, d t)$ for each $\lambda>0$. Let $g \in L^{2}(I, E, d t)$. Then, from $(H 3) t \mapsto v(t)=$

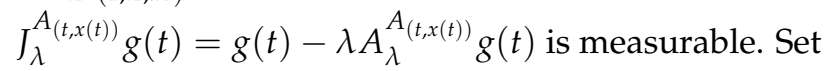

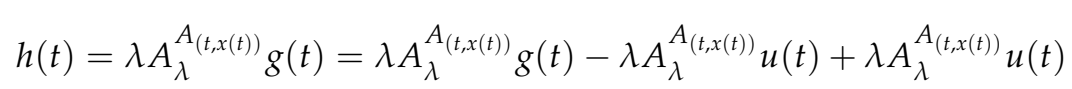

where $u$ denotes the absolutely continuous solution to $-\frac{d u}{d t}(t) \in A_{(t, x(t))} u(t)$ using Fact $\mathcal{I}$. Then, $h$ is measurable with

$$
\|h(t)\| \leq 2\|g(t)-u(t)\|+\lambda\left\|A_{\lambda}^{A_{(t, x(t))}} u(t)\right\|
$$

by noting that $A_{\lambda}^{A_{(t, x(t))}}$ is $\frac{2}{\lambda}$-Lipschitz and so we deduce that $h \in L^{2}(I, E, d t)$ because $g \in L^{2}(I, E, d t)$ and $t \mapsto A_{\lambda}^{A_{(t, x(t))}} u(t) \in L^{\infty}(I, E, d t)$ using $(H 1)$. This proves that $v \in L^{2}(I, E, d t)$ and $g \in v+\lambda \mathcal{A}_{x} v$ so that $R\left(I_{L^{2}(I, E ; d t)}+\lambda \mathcal{A}_{x}\right)=L^{2}(I, E, d t)$.

Here is a useful application.

Corollary 1. With hypotheses and notation of the preceding lemma, let $\left(v_{n}\right)$ and $\left(u_{n}\right)$ be two sequences in $L^{2}(I, E, d t)$ such that $v_{n}(t) \in A_{(t, x(t))} u_{n}(t)$ a.e for all $n \in \mathbf{N}$. If $v_{n} \rightarrow v$ weakly in $L^{2}(I, E, d t)$ and $u_{n} \rightarrow u$ strongly in $L^{2}(I, E, d t)$, then $v(t) \in A_{(t, x(t))} u(t)$ a.e.

Theorem 1. Let $I=[0, T]$. Let $(t, x) \rightarrow A_{(t, x)}: D\left(A_{(t, x)}\right) \rightarrow 2^{E}$ a maximal monotone operator satisfying: $\left(H_{1}\right)\left\|A_{(t, x)}^{0} y\right\| \leq c(1+\|x\|+\|y\|)$ for all $(t, x, y) \in I \times E \times D\left(A_{(t, x)}\right)$, for some positive constant $c$,

$\left(H_{2}\right) \operatorname{dis}\left(A_{(t, x)}, A_{(\tau, y)}\right) \leq a(t)-a(\tau)+r\|x-y\|$, for all $0 \leq \tau \leq t \leq T$ and for all $(x, y) \in E \times E$, where $r$ is a positive number, $a: I \rightarrow\left[0,+\infty\left[\right.\right.$ is nondecreasing absolutely continuous on $I$ with $\dot{a} \in L^{2}(I, \mathbb{R}, d t)$, $\left(H_{3}\right) D\left(A_{(t, x)}\right)$ is boundedly-compactly measurable in the sense, for any bounded set $B \subset E$, there is a measurable compact valued integrably bounded mapping $\Psi_{B}: I \rightarrow E$ such that $D\left(A_{(t, x)}\right) \subset \Psi_{B}(t) \subset \gamma(t) \bar{B}_{E}$ for all $(t, x) \in I \times B$ where $\gamma \in L^{2}(I, \mathbb{R}, d t)$.

Then, for any $\left(x_{0}, u_{0}\right) \in E \times D\left(A_{\left(0, x_{0}\right)}\right)$, there exist an absolutely continuous $x: I \rightarrow E$ and an absolutely continuous $u: I \rightarrow$ Esuch that

$$
\left\{\begin{array}{l}
x(t)=x_{0}+\int_{0}^{t} u(s) d s, \quad \forall t \in I \\
x(0)=x_{0}, u(0)=u_{0} \in D\left(A_{\left(0, x_{0}\right)}\right) \\
-\dot{u}(t) \in A_{(t, x(t))} u(t) \quad \text { a.e. } t \in I \\
u(t) \in D\left(A_{(t, x(t))}\right), \forall t \in I
\end{array}\right.
$$

Proof. Let us consider the closed convex subset $\mathcal{X}_{\gamma}$ in the Banach space $\mathcal{C}_{E}(I)$ defined by

$$
\mathcal{X}_{\gamma}:\left\{h \in W^{1,2}(I, E): h(t)=x_{0}+\int_{0}^{t} \dot{h}(s) d s,\|\dot{h}(s)\| \leq \gamma(s) \text { a.e., } \gamma \in L^{2}(I, \mathbf{R}, d t)\right\} .
$$


Then, $\mathcal{X}_{\gamma}$ is equi-absolutely continuous. By the fact that $\mathcal{J}$, for each $h \in X_{\gamma}$, there is a unique $W^{1,2}(I, E)$ mapping $u_{h}: I \rightarrow E$, which is the $W^{1,2}(I, E)$ solution to the inclusion

$$
\left\{\begin{array}{l}
-\dot{u}_{h}(t) \in A_{(t, h(t))} u_{h}(t) \quad \text { a.e. } t \in I \\
u_{h}(t) \in D\left(A_{(t, h(t))}\right), \forall t \in I \\
u_{h}(0)=u_{0} \in D\left(A_{(0, h(0))}\right)=D\left(A_{\left.\left(0, x_{0}\right)\right)}\right)
\end{array}\right.
$$

with $\left\|\dot{u}_{h}(t)\right\| \leq K(1+\dot{\beta}(t))$, where $\beta(t)=\int_{0}^{t}[\dot{a}(s)+\gamma(s)] d s, \forall t \in I$ and $K$ is a positive constant depending on $\left\|u_{0}\right\|, c, T, \beta$. We refer to [22] (Theorem 3.5) for details of the estimate of the velocity. Now, for each $h \in \mathcal{X}_{\gamma}$, let us consider the mapping

$$
\Phi(h)(t):=x_{0}+\int_{0}^{t} u_{h}(s) d s, t \in I .
$$

As $u_{h}(s) \in D\left(A_{(s, h(s))}\right) \subset \bigcup_{x \in \mathcal{X}_{\gamma}(s)} D\left(A_{(s, x)}\right) \subset \Psi_{\gamma}(s) \subset \gamma(s) \bar{B}_{E}$ for all $s \in[0, T]$, where $\Psi_{\gamma}$ : $I \rightarrow E$ is a compact valued measurable mapping given by condition $\left(H_{3}\right)$. It is clear that $\Phi(h) \in \mathcal{X}_{\gamma}$. Our aim is to prove the existence theorem by applying some ideas developed in [24] via a generalized fixed point theorem [25] (Theorem 4.3), [26] (Lemma 1). Nevertheless, this needs a careful look using the estimation of the absolutely continuous solution given above. For this purpose, we first claim that $\Phi: \mathcal{X}_{\gamma} \rightarrow \mathcal{X}_{\gamma}$ is continuous and, for any $h \in \mathcal{X}_{\gamma}$ and for any $t \in I$, the inclusion holds

$$
\Phi(h)(t) \in u_{0}+\int_{0}^{t} \overline{c o} \Psi_{\gamma}(s) d s .
$$

Since $s \mapsto \overline{c o} \Psi_{\gamma}(s)$ is a convex compact valued and integrably bounded multifunction, the second member is convex compact valued [27] so that $\Phi(\mathcal{X})$ is equicontinuous and relatively compact in the Banach space $\mathcal{C}_{E}(I)$. Now, we check that $\Phi$ is continuous. It is sufficient to show that, if $\left(h_{n}\right)$ converges uniformly to $h$ in $\mathcal{X}_{\gamma}$, then the AC solution $u_{h_{n}}$ associated with $h_{n}$

$$
\left\{\begin{array}{r}
u_{h_{n}}(0) \in D\left(A_{\left(0, h_{n}(0)\right)}\right) \\
u_{h_{n}}(t) \in D\left(A_{\left(t, h_{n}(t)\right)}\right), \forall t \in I \\
-\dot{u}_{h_{n}}(t) \in A_{\left(t, h_{h}(t)\right)} u_{h_{n}}(t) \quad \text { a.e. } t \in I
\end{array}\right.
$$

uniformly converges to the AC solution $u_{h}$ associated with $h$

$$
\left\{\begin{array}{r}
u_{h}(0)=u_{0} \in D\left(A_{(0, h(0))}\right) \\
u_{h}(t) \in D\left(A_{(t, h(t))}\right), \forall t \in I \\
-\dot{u}_{h}(t) \in A_{(t, h(t))} u_{h}(t) \quad \text { a.e. } t \in I
\end{array}\right.
$$

As $\left(u_{h_{n}}\right)$ is equi-absolutely continuous with the estimate $\left\|\dot{u}_{h_{n}}(t)\right\| \leq K(1+\dot{\beta}(t))$ a.e for all $n \in \mathbf{N}$, we may assume that $\left(u_{h_{n}}\right)$ converges uniformly to a AC mapping $u$ and $\left(\frac{d u_{h_{n}}}{d t}\right)$ converges weakly in $L_{E}^{2}(I, d t)$ to $w \in L_{E}^{2}(I, d t)$ with $\|w(t)\| \leq K(1+\dot{\beta}(t))$ a.e. $t \in I$ so that

$$
\begin{aligned}
& \text { weak- } \lim _{n} u_{h_{n}}=\text { weak- } \lim _{n} u_{h_{n}}(0)+\text { weak- } \lim _{n} \int_{I} \frac{d u_{h_{n}}}{d t} \\
& =u(0)+\int_{I} w d t:=z(t), t \in I
\end{aligned}
$$


By identifying the limits, we get $u(t)=z(t)=u(0)+\int_{I} w d t, t \in I$ with $u(0)=$ weak- $\lim _{n} u_{h_{n}}(0)=\lim _{n} u_{h_{n}}(0)$ and $\frac{d u}{d t}=w$. As $u_{h_{n}}(t) \in D\left(A_{\left(t, h_{n}(t)\right)}\right), \forall t \in I$ and $u_{h_{n}}(t) \rightarrow u(t)$, $A_{\left(t, h_{n}(t)\right)}^{0} u_{h_{n}}(t)$ is bounded using $\left(H_{1}\right)$ for every $t \in[0, T]$ and

$$
\operatorname{dis}\left(A_{\left(t, h_{n}(t)\right)}, A_{(t, h(t))}\right) \leq r\left\|h_{n}(t)-h(t)\right\| \rightarrow 0
$$

when $n \rightarrow \infty$ by $\left(H_{2}\right)$, from Lemma 2, we deduce that $u(t) \in D\left(A_{(t, h(t))}\right), \forall t \in I$. Now, we are going to check that $u$ satisfies the inclusion

$$
-\frac{d u}{d t}(t) \in A_{(t, h(t))} u(t) \quad \text { a.e. } t \in I
$$

As $\frac{d u_{h_{n}}}{d t} \rightarrow \frac{d u}{d t}$ weakly in $L^{2}(I, E, d t)$, we may assume that $\left(\frac{d u_{h_{n}}}{d t}\right)$ Komlos converges to $\frac{d u}{d t}$. There is a $d t$-negligible set $N$ such that for $t \in I \backslash N$

$$
\begin{aligned}
& \lim _{n \rightarrow \infty} \frac{1}{n} \sum_{j=1}^{n} \frac{d u_{h_{j}}}{d t}(t)=\frac{d u}{d t}(t) . \\
& -\frac{d u_{h_{n}}}{d t}(t) \in A_{\left(t, h_{n}(t)\right)} u_{n}(t) .
\end{aligned}
$$

Let $\eta \in D\left(A_{(t, h(t))}\right)$.

Using Lemma 4 , there is a sequence $\left(\eta_{n}\right)$ such that $\eta_{n} \in D\left(A_{\left(t, h_{n}(t)\right)}\right), \eta_{n} \rightarrow \eta$ and $A_{\left(t, h_{n}(t)\right)}^{0} \eta_{n} \rightarrow$ $A_{(t, h(t))}^{0} \eta$. From (4), by monotonicity,

$$
\left\langle\frac{d u_{h_{n}}}{d t}, u_{h_{n}}(t)-\eta_{n}\right\rangle \leq\left\langle A_{\left(t, h_{n}(t)\right)}^{0} \eta_{n}, \eta_{n}-u_{h_{n}}(t)\right\rangle .
$$

From

$$
\left\langle\frac{d u_{h_{n}}}{d t}(t), u(t)-\eta\right\rangle=\left\langle\frac{d u_{h_{n}}}{d t}(t), u_{h_{n}}(t)-\eta_{n}\right\rangle+\left\langle\frac{d u_{h_{n}}}{d t}(t), u(t)-u_{h_{n}}(t)-\left(\eta-\eta_{n}\right)\right\rangle,
$$

let us write

$$
\begin{gathered}
\frac{1}{n} \sum_{j=1}^{n}\left\langle\frac{d u_{h_{j}}}{d t}(t), u(t)-\eta\right\rangle=\frac{1}{n} \sum_{j=1}^{n}\left\langle\frac{d u_{h_{j}}}{d t}(t), u_{h_{j}}(t)-\eta_{j}\right\rangle+\frac{1}{n} \sum_{j=1}^{n}\left\langle\frac{d u_{h_{j}}}{d t}(t), u(t)-u_{h_{j}}(t)\right\rangle \\
+\sum_{j=1}^{n}\left\langle\frac{d u_{h_{j}}}{d t}(t), \eta_{j}-\eta\right\rangle,
\end{gathered}
$$

so that

$$
\begin{gathered}
\left.\frac{1}{n} \sum_{j=1}^{n}\left\langle\frac{d u_{h_{j}}}{d t}(t), u(t)-\eta\right\rangle \leq \frac{1}{n} \sum_{j=1}^{n}\left\langle A_{\left(t, h_{j}(t)\right)}^{0} \eta_{j}, \eta_{j}-u_{h_{j}}(t)\right\rangle+K(1+\dot{\beta}(t)) \frac{1}{n} \sum_{j=1}^{n} \| u(t)-u_{h_{j}}(t)\right) \| . \\
+K(1+\dot{\beta}(t)) \frac{1}{n} \sum_{j=1}^{n}\left\|\eta_{j}-\eta\right\| .
\end{gathered}
$$

Passing to the limit using (3) when $n \rightarrow \infty$, this last inequality gives immediately

$$
\left\langle\frac{d u}{d t}(t), u(t)-\eta\right\rangle \leq\left\langle A_{(t, h(t))}^{0} \eta, \eta-u(t)\right\rangle \text { a.e. }
$$


As a consequence, by Lemma 1 , we get $-\frac{d u}{d t}(t) \in A_{(t, h(t))} u(t)$ a.e. with $u(0) \in D\left(A_{(0, h(0))}\right)$ so that, by uniqueness, $u=u_{h}$.

Now, let us check that $\Phi: \mathcal{X} \rightarrow \mathcal{X}$ is continuous. Let $h_{n} \rightarrow h$. We have

$$
\Phi\left(h_{n}\right)(t)-\Phi(h)(t)=\int_{0}^{t} u_{h_{n}}(s) d s-\int_{0}^{t} u_{h}(s) d s=\int_{0}^{t}\left[u_{h_{h}}(s)-u_{h}(s)\right] d s
$$

As $\left\|u_{h_{n}}()-.u_{h}().\right\| \rightarrow 0$ pointwisely and is uniformly bounded, we conclude that

$$
\sup _{t \in I}\left\|\Phi\left(h_{n}\right)(t)-\Phi(h)(t)\right\| \leq \sup _{t \in I} \int_{0}^{t}\left\|u_{h_{n}}(.)-u_{h}(.)\right\| d s \rightarrow 0
$$

so that $\Phi\left(h_{n}\right)-\Phi(h) \rightarrow 0$ in $\mathcal{C}_{E}(I)$. Since $\Phi: \mathcal{X}_{\gamma} \rightarrow \mathcal{X}_{\gamma}$ is continuous and $\Phi\left(\mathcal{X}_{\gamma}\right)$ is relatively compact in $\mathcal{C}_{E}(I)$, by [25] (Theorem 4.3), [26] (Lemma 1), $\Phi$ has a fixed point, say $h=\Phi(h) \in \mathcal{X}_{\gamma}$ that means

$$
\begin{gathered}
h(t)=\Phi(h)(t)=x_{0}+\int_{0}^{t} u_{h}(s) d s, t \in I, \\
\left\{\begin{array}{l}
u_{h}(t) \in D\left(A_{(t, h(t))}\right) \\
-\frac{d u_{h}}{d t}(t) \in A_{(t, h(t))} u_{h}(t) d t \text {-a.e. }
\end{array}\right.
\end{gathered}
$$

the proof is complete.

There is a direct application to sweeping process.

Corollary 2. Let $C: I \times E \rightarrow E$ be a convex compact valued mapping satisfying

(i) $C(t, x) \subset \gamma(t) \bar{B}_{E}, \forall(t, x) \in I \times E$, where $\gamma \in L^{2}(I, \mathbb{R}, d t)$,

(ii) $d_{H}(C(s, x), C(t, y)) \leq a(t)-a(\tau)+r\|x-y\|$, for all $0 \leq \tau \leq t \leq 1$ and for all $(x, y) \in E \times E$, where $r$ is a positive number, $a: I \rightarrow\left[0,+\infty\left[\right.\right.$ is nondecreasing absolutely continuous on $I$ with $\dot{a} \in L^{2}(I, \mathbb{R}, d t)$,

(iii) For any $t \in I$, for any bounded set $B \subset E, C(t, B)$ is relatively compact.

Then, for any $\left(x_{0}, u_{0}\right) \in E \times C\left(0, x_{0}\right)$, there exist an absolutely continuous $x: I \rightarrow E$ and and absolutely continuous $u: I \rightarrow$ Esuch that

$$
\left\{\begin{array}{l}
x(t)=x_{0}+\int_{0}^{t} u(s) d s, \quad \forall t \in I \\
x(0)=x_{0}, u(0)=u_{0} \in C\left(0, x_{0}\right) \\
-\dot{u}(t) \in N_{C(t, x(t))} u(t) \quad \text { a.e. } t \in I \\
u(t) \in C(t, x(t)), \forall t \in I
\end{array}\right.
$$

Proof. It is easy to apply Theorem 1 with $A_{(t, x(t))}=N_{C(t, x(t))}$

Now, we proceed to the Lipschitz perturbation of the preceding theorem.

Theorem 2. Let $I=[0, T]$. Let $(t, x) \rightarrow A_{(t, x)}: D\left(A_{(t, x)}\right) \rightarrow 2^{E}$ be a maximal monotone operator satisfying: $\left(H_{1}\right)\left\|A_{(t, x)}^{0} y\right\| \leq c(1+\|x\|+\|y\|)$ for all $(t, x, y) \in I \times E \times D\left(A_{(t, x)}\right)$, for some positive constant $c$, $\left(H_{2}\right) \operatorname{dis}\left(A_{(t, x)}, A_{(\tau, y)}\right) \leq a(t)-a(\tau)+r\|x-y\|$, for all $0 \leq \tau \leq t \leq T$ and for all $(x, y) \in E \times E$, where $r$ is a positive number, $a: I \rightarrow\left[0,+\infty\left[\right.\right.$ is nondecreasing absolutely continuous on $I$ with $\dot{a} \in L^{2}(I, \mathbb{R}, d t)$,

$\left(H_{3}\right) D\left(A_{(t, x)}\right)$ is boundedly-compactly measurable in the sense, for any bounded set $B \subset E$, there is a measurable compact valued integrably bounded mapping $\Psi_{B}: I \rightarrow$ E such that $D\left(A_{(t, x)}\right) \subset \Psi_{B}(t) \subset \gamma(t) \bar{B}_{E}$ for all $(t, x) \in I \times B$, where $\gamma \in L^{2}(I, \mathbb{R}, d t)$.

Let $f: I \times E \times E \rightarrow E$ such that

(i) $f(., x, y)$ is Lebesgue measurable on I for all $(x, y) \in E \times E$

(ii) $f(t, . .$.$) is continuous on E \times E$,

(iii) $\|f(t, x, y)\| \leq M$ for all $(t, x, y) \in I \times E \times E$,

(iv) $\|f(t, x, y)-f(t, x, z)\| \leq M\|y-z\|$, for all $(t, x, y, z) \in I \times E \times E \times E$ 
for some positive constant $M$.

Then, for any $\left(x_{0}, u_{0}\right) \in E \times D\left(A_{\left(0, x_{0}\right)}\right)$, there exists an absolutely continuous $x: I \rightarrow E$ and an absolutely continuous $u: I \rightarrow$ Esuch that

$$
\left\{\begin{array}{l}
x(t)=x_{0}+\int_{0}^{t} u(s) d s, \quad \forall t \in I \\
x(0)=x_{0}, u(0)=u_{0} \in D\left(A_{\left(0, x_{0}\right)}\right) \\
-\dot{u}(t) \in A_{(t, x(t))} u(t)+f(t, x(t), u(t)) \quad \text { a.e. } t \in I \\
u(t) \in D\left(A_{(t, x(t))}\right), \forall t \in I
\end{array}\right.
$$

Proof. Let us consider the closed convex subset $\mathcal{X}_{\gamma}$ in the Banach space $\mathcal{C}_{E}(I)$ defined by

$$
\mathcal{X}_{\gamma}:\left\{h \in W^{1,2}(I, E): h(t)=x_{0}+\int_{0}^{t} \dot{h}(s) d s,\|\dot{h}(s)\| \leq \gamma(s) \text { a.e., } \gamma \in L^{2}(I, \mathbf{R}, d t)\right\} \text {. }
$$

Then, $\mathcal{X}_{\gamma}$ is equi-absolutely continuous. By fact $\mathcal{J}$, for each $h \in X_{\gamma}$, there is a unique $W^{1,2}(I, E)$ mapping $u_{h}: I \rightarrow E$, which is the $W^{1,2}(I, E)$ solution to the inclusion

$$
\left\{\begin{array}{l}
-\dot{u}_{h}(t) \in A_{(t, h(t))} u_{h}(t)+f\left(t, h(t), u_{h}(t)\right) \quad \text { a.e. } t \in I \\
u_{h}(t) \in D\left(A_{(t, h(t))}\right), \forall t \in I \\
u_{h}(0)=u_{0} \in D\left(A_{(0, h(0))}\right)=D\left(A_{\left(0, x_{0}\right)}\right)
\end{array}\right.
$$

with $\left\|\dot{u}_{h}(t)\right\| \leq K(1+\dot{\beta}(t))+M(K+1)=\eta(t)$ where $\beta(t)=\int_{0}^{t}[\dot{a}(s)+\gamma(s)] d s, \forall t \in I$ and $K$ is a positive constant depending on $\left\|u_{0}\right\|, c, T, \beta$. We refer to (Theorem 3.5) for details of the estimate of the velocity. Now, for each $h \in \mathcal{X}_{\gamma}$, let us consider the mapping

$$
\Phi(h)(t):=x_{0}+\int_{0}^{t} u_{h}(s) d s, t \in I
$$

As $u_{h}(s) \in D\left(A_{(s, h(s))}\right) \subset \bigcup_{x \in \mathcal{X}_{\gamma}(s)} D\left(A_{(s, x)}\right) \subset \Psi_{\gamma}(s) \subset \gamma(s) \bar{B}_{E}$ for all $s \in[0, T]$, where $\Psi_{\gamma}:$ $I \rightarrow E$ is a compact valued measurable mapping given by condition $\left(H_{3}\right)$. It is clear that $\Phi(h) \in \mathcal{X}_{\gamma}$. Our aim is to prove the existence theorem by applying some ideas developed in Castaing et al. [24] via the same generalized fixed point theorem already used $[25,26]$. Nevertheless, this needs a careful look using the estimation of the absolutely continuous solution given above. For this purpose, we first claim that $\Phi: \mathcal{X}_{\gamma} \rightarrow \mathcal{X}_{\gamma}$ is continuous, and, for any $h \in \mathcal{X}_{\gamma}$ and for any $t \in I$, the inclusion holds

$$
\Phi(h)(t) \in u_{0}+\int_{0}^{t} \overline{c o} \Psi_{\gamma}(s) d s
$$

Since $s \mapsto \overline{C O} \Psi_{\gamma}(s)$ is a convex compact valued and integrably bounded multifunction, the second member is convex compact valued [27] so that $\Phi(\mathcal{X})$ is equicontinuous and relatively compact in the Banach space $\mathcal{C}_{E}(I)$. Now, we check that $\Phi$ is continuous. It is sufficient to show that, if $\left(h_{n}\right)$ converges uniformly to $h$ in $\mathcal{X}_{\gamma}$, then the AC solution $u_{h_{n}}$ associated with $h_{n}$

$$
\left\{\begin{array}{r}
u_{h_{n}}(0) \in D\left(A_{\left(0, h_{n}(0)\right)}\right) \\
u_{h_{n}}(t) \in D\left(A_{\left(t, h_{n}(t)\right)}\right), \forall t \in I \\
-\dot{u}_{h_{n}}(t) \in A_{\left(t, h_{n}(t)\right)} u_{h_{n}}(t)+f\left(t, h_{n}(t), u_{h_{n}}(t)\right), \quad \text { a.e. } t \in I
\end{array}\right.
$$

uniformly converges to the AC solution $u_{h}$ associated with $h$

$$
\left\{\begin{array}{r}
u_{h}(0) \in D\left(A_{(0, h(0))}\right) \\
u_{h}(t) \in D\left(A_{(t, h(t))}\right), \forall t \in I \\
-\dot{u}_{h}(t) \in A_{(t, h(t))} u_{h}(t)+f\left(t, h(t), u_{h}(t)\right) \text { a.e. } t \in I
\end{array}\right.
$$


As $\left(u_{h_{n}}\right)$ is equi-absolutely continuous with the estimate $\left\|\dot{u}_{h_{n}}(t)\right\| \leq K(1+\dot{\beta}(t))+(K+1) M=$ $\psi(t)$ a.e for all $n \in \mathbf{N}$, we may assume that $\left(u_{h_{n}}\right)$ converges uniformly to a AC mapping $u$ and $\left(\frac{d u_{h_{n}}}{d t}\right)$ converges weakly in $L_{E}^{2}(I, d t)$ to $w \in L_{E}^{2}(I, d t)$ with $\|w(t)\| \leq K(1+\dot{\beta}(t))+(K+1) M$ a.e. $t \in I$ so that

$$
\begin{gathered}
\text { weak- } \lim _{n} u_{h_{n}}=\text { weak- } \lim _{n} u_{h_{n}}(0)+\text { weak- } \lim _{n} \int_{[0, t]} \frac{d u_{h_{n}}}{d t} \\
=u(0)+\int_{[0, t]} w d t:=z(t), t \in I
\end{gathered}
$$

By identifying the limits, we get

$u(t)=z(t)=u(0)+\int_{[0, t]} w d t, t \in I$ with $u(0)=$ weak- $\lim _{n} u_{h_{n}}(0)=\lim _{n} u_{h_{n}}(0)$ and $\frac{d u}{d t}=w$. As $u_{h_{n}}(t) \in D\left(A_{\left(t, h_{n}(t)\right)}\right), \forall t \in I$ and $u_{h_{n}}(t) \rightarrow u(t), A_{\left(t, h_{n}(t)\right)}^{0} u_{h_{n}}(t)$ is bounded using $\left(H_{1}\right)$ for every $t \in I$ and

$$
\operatorname{dis}\left(A_{\left(t, h_{n}(t)\right.}, A_{(t, h(t)}\right) \leq r|| h_{n}(t)-h(t) \| \rightarrow 0
$$

when $n \rightarrow \infty$ by $\left(H_{2}\right)$, from Lemma 2, we deduce that $u(t) \in D\left(A_{(t, h(t))}\right), \forall t \in I$.

Now, we are going to check that $u$ satisfies the inclusion

$$
-\frac{d u}{d t}(t) \in A_{(t, h(t))} u(t)+f\left(t, h(t), u_{h}(t)\right) \quad \text { a.e. } t \in I
$$

As $\dot{u}_{h_{n}} \rightarrow \dot{u}$ weakly in $L_{H}^{2}([0,1]), \dot{u}_{h_{n}} \rightarrow \dot{u}$ Komlos. Note that $f\left(t, h_{n}(t), u_{h_{n}}(t)\right) \rightarrow f(t, h(t), u(t))$ weakly in $L_{E}^{2}([0,1])$. Thus, $z_{n}(t):=f\left(t, h_{n}(t), u_{h_{n}}(t)\right) \rightarrow z(t):=f(t, h(t), u(t))$ Komlos. Hence, $\dot{u}_{h_{n}}(t)+f\left(t, h_{n}(t), u_{h_{n}}(t) \rightarrow \dot{u}(t)+f(t, h(t), u(t))\right.$ Komlos. Apply Lemma 4 to $A_{\left(t, h_{n}(t)\right)}$ and $\left.A_{(t, h(t)}\right)$ to find a sequence $\left(\eta_{n}\right)$ such that $\eta_{n} \in D\left(A_{\left(t, h_{n}(t)\right)}\right), \eta_{n} \rightarrow \eta, A_{\left(t, h_{n}(t)\right.}^{0} \eta_{n} \rightarrow A_{(t, h(t))}^{0} u(t)$. From

$$
-\dot{u}_{h_{n}}(t) \in A_{\left(t, h_{n}(t)\right)} u_{h_{n}}(t)+f\left(t, h_{n}(t), u_{h_{n}}(t)\right)
$$

by monotonicity

$$
\left.\left\langle\frac{d u_{h_{n}}}{d t}+z_{n}(t), u_{h_{n}}(t)-\eta_{n}\right\rangle \leq A_{\left(t, h_{n}(t)\right)}^{0} \eta_{n}, \eta_{n}-u_{h_{n}}(t)\right\rangle .
$$

From

$$
\begin{gathered}
\left\langle\frac{d u_{h_{n}}}{d t}(t)+z_{n}(t), u(t)-\eta\right\rangle=\left\langle\frac{d u_{h_{n}}}{d t}(t)+z_{n}(t), u_{h_{n}}(t)-\eta_{n}\right\rangle \\
+\left\langle\frac{d u_{h_{n}}}{d t}(t)+z_{n}(t), u(t)-u_{h_{n}}(t)-\left(\eta-\eta_{n}\right)\right\rangle,
\end{gathered}
$$

let us write

$$
\begin{gathered}
\frac{1}{n} \sum_{j=1}^{n}\left\langle\frac{d u_{h_{j}}}{d t}(t)+z_{j}(t), u(t)-\eta\right\rangle=\frac{1}{n} \sum_{j=1}^{n}\left\langle\frac{d u_{h_{j}}}{d t}(t)+z_{j}(t), u_{h_{j}}(t)-\eta_{j}\right\rangle \\
+\frac{1}{n} \sum_{j=1}^{n}\left\langle\frac{d u_{h_{j}}}{d t}(t)+z_{j}(t), u(t)-u_{h_{j}}(t)\right\rangle \\
+\sum_{j=1}^{n}\left\langle\frac{d u_{h_{j}}}{d t}(t)+z_{j}(t), \eta_{j}-\eta\right\rangle,
\end{gathered}
$$

so that

$$
\left.\frac{1}{n} \sum_{j=1}^{n}\left\langle\frac{d u_{h_{j}}}{d t}(t)+z_{j}(t), u(t)-\eta\right\rangle \leq \frac{1}{n} \sum_{j=1}^{n}\left\langle A_{\left(t, h_{j}(t)\right)}^{0} \eta_{j}, \eta_{j}-u_{h_{j}}(t)\right\rangle+(\psi(t)+M) \frac{1}{n} \sum_{j=1}^{n} \| v(t)-u_{h_{j}}(t)\right) \| .
$$




$$
+(\psi(t)+M) \frac{1}{n} \sum_{j=1}^{n}\left\|\eta_{j}-\eta\right\|
$$

Passing to the limit using (3) when $n \rightarrow \infty$, this last inequality gives immediately

$$
\left\langle\frac{d u}{d t}(t)+z(t), u(t)-\eta\right\rangle \leq\left\langle A_{(t, h(t))}^{0} \eta, \eta-u(t)\right\rangle \text { a.e. }
$$

As a consequence, by Lemma 1 , we get $-\frac{d u}{d t}(t) \in A_{(t, h(t))} u(t)+z(t)$ a.e. with $u(t) \in D\left(A_{(t, h(t))}\right)$ for all $t \in[0,1]$ so that, by uniqueness, $u=u_{h}$.

Since $h_{n} \rightarrow h$, we have

$$
\begin{gathered}
\Phi\left(h_{n}\right)(t)-\Phi(h)(t)=\int_{0}^{1} u_{h_{n}}(s) d s-\int_{0}^{1} u_{h}(s) d s \\
=\int_{0}^{1}\left[u_{h_{n}}(s)-u_{h}(s)\right] d s \\
\leq \int_{0}^{1}\left\|u_{h_{n}}(s)-u_{h}(s)\right\| d s
\end{gathered}
$$

As $\left\|u_{h_{n}}(\cdot)-u_{h}(\cdot)\right\| \rightarrow 0$ uniformly, we conclude that

$$
\sup _{t \in[0,1]}\left\|\Phi\left(h_{n}\right)(t)-\Phi(h)(t)\right\| \leq \int_{0}^{1}\left\|u_{h_{n}}(\cdot)-u_{h}(\cdot)\right\| d s \rightarrow 0
$$

so that $\Phi\left(h_{n}\right) \rightarrow \Phi(h)$ in $\mathcal{C}_{E}([0,1])$. Since $\Phi: \mathcal{X}_{\gamma} \rightarrow \mathcal{X}_{\gamma}$ is continuous and $\Phi\left(\mathcal{X}_{\gamma}\right)$ is relatively compact in $\mathcal{C}_{E}(I)$, by $[25,26] \Phi$ has a fixed point, say $h=\Phi(h) \in \mathcal{X}_{\gamma}$ that means

$$
\begin{gathered}
h(t)=\Phi(h)(t)=x_{0}+\int_{0}^{t} u_{h}(s) d s, t \in I, \\
\left\{\begin{array}{l}
u_{h}(t) \in D\left(A_{(t, h(t))}\right) \\
-\frac{d u_{h}}{d t}(t) \in A_{(t, h(t))} u_{h}(t)+f\left(t, h(t), u_{h}(t)\right) d t \text {-a.e. }
\end{array}\right.
\end{gathered}
$$

The proof is complete.

\section{Towards a Fractional Order of Evolution Inclusion with a Time and State Dependent Maximal Monotone Operator}

Now, $I=[0,1]$ and we investigate a class of boundary value problem governed by a fractional differential inclusion (FDI) in a separable Hilbert space $E$ coupled with an evolution inclusion governed by a time and stated dependent maximal monotone operator:

$$
\begin{gathered}
D^{\alpha} h(t)+\lambda D^{\alpha-1} h(t)=u(t), t \in I, \\
\left.I_{0^{+}}^{\beta} h(t)\right|_{t=0}:=\lim _{t \rightarrow 0} \int_{0}^{t} \frac{(t-s)^{\beta-1}}{\Gamma(\beta)} h(s) d s=0, \quad h(1)=I_{0^{+}}^{\gamma} h(1)=\int_{0}^{1} \frac{(1-s)^{\gamma-1}}{\Gamma(\gamma)} h(s) d s, \\
-\frac{d u}{d t}(t) \in A_{(t, h(t))} u(t) \text { a.e. } t \in I .
\end{gathered}
$$

where $\alpha \in] 1,2], \beta \in[0,2-\alpha], \lambda \geq 0, \gamma>0$ are given constants, $D^{\alpha}$ is the standard Riemann-Liouville fractional derivative, and $\Gamma$ is the gamma function. 


\subsection{Fractional Calculus}

For the convenience of the reader, we begin with a few reminders of the concepts that will be used in the rest of the paper.

Definition 1 (Fractional Bochner integral). Let $E$ be a separable Banach space. Let $f: I=[0,1] \rightarrow E$. The fractional Bochner-integral of order $\alpha>0$ of the function $f$ is defined by

$$
I_{a^{+}}^{\alpha} f(t):=\int_{a}^{t} \frac{(t-s)^{\alpha-1}}{\Gamma(\alpha)} f(s) d s, t>a .
$$

In the above definition, the sign " $\int$ " denotes the classical Bochner integral.

Lemma 6 ([10]). Let $f \in L^{1}([0,1], E, d t)$. We have

(i) If $\alpha \in] 0,1\left[\right.$ then $I^{\alpha} f$ exists almost everywhere on $I$ and $I^{\alpha} f \in L^{1}(I, E, d t)$.

(ii) If $\alpha \in[1, \infty)$, then $I^{\alpha} f \in C_{E}(I)$.

Definition 2. Let $E$ be a separable Banach space. Let $f \in L^{1}(I, E, d t)$. We define the Riemann-Liouville fractional derivative of order $\alpha>0$ of $f$ by

$$
D^{\alpha} f(t):=D_{0^{+}}^{\alpha} f(t)=\frac{d^{n}}{d t^{n}} I_{0^{+}}^{n-\alpha} f(t)=\frac{d^{n}}{d t^{n}} \int_{0}^{t} \frac{(t-s)^{n-\alpha-1}}{\Gamma(n-\alpha)} f(s) d s,
$$

where $n=[\alpha]+1$.

In the case $E \equiv \mathbf{R}$, we have the following well-known results.

Lemma $7([1,3])$. Let $\alpha>0$. The general solution of the fractional differential equation $D^{\alpha} x(t)=0$ is given by

$$
x(t)=c_{1} t^{\alpha-1}+c_{2} t^{\alpha-2}+\cdots+c_{N} t^{\alpha-N},
$$

where $c_{i} \in \mathbf{R}, i=1,2, \ldots, N$ ( $N$ is the smallest integer greater than or equal to $\alpha$ ).

Remark 1. Since $D_{0^{+}}^{\alpha} I_{0^{+}}^{\alpha} v(t)=v(t)$, for every $v \in C(I), D_{0^{+}}^{\alpha}\left[I_{0^{+}}^{\alpha} D_{0^{+}}^{\alpha} x(t)-x(t)\right]=0$ and, by Lemma 7, it follows that

$$
x(t)=I_{0^{+}}^{\alpha} D_{0^{+}}^{\alpha} x(t)+c_{1} t^{\alpha-1}+\cdots+c_{N} t^{\alpha-N},
$$

for some $c_{i} \in \mathbf{R}, i=1,2, \ldots, N$.

We denote by $W_{B, E}^{\alpha, 1}(I)$ the space of all continuous functions in $C_{E}(I)$ such that their Riemann-Liouville fractional derivative of order $\alpha-1$ are continuous and their Riemann-Liouville fractional derivative of order $\alpha$ are Bochner integrable.

\subsection{Green Function and Its Properties}

Let $\alpha \in] 1,2], \beta \in[0,2-\alpha], \lambda \geq 0, \gamma>0$ and $G:[0,1] \times[0,1] \rightarrow \mathbf{R}$ be a function defined by

$$
G(t, s)=\varphi(s) I_{0^{+}}^{\alpha-1}(\exp (-\lambda t))+ \begin{cases}\exp (\lambda s) I_{s^{+}}^{\alpha-1}(\exp (-\lambda t)), & 0 \leq s \leq t \leq 1 \\ 0, & 0 \leq t \leq s \leq 1\end{cases}
$$


where

$$
\varphi(s)=\frac{\exp (\lambda s)}{\mu_{0}}\left[\left(I_{s^{+}}^{\alpha-1+\gamma}(\exp (-\lambda t))\right)(1)-\left(I_{s^{+}}^{\alpha-1}(\exp (-\lambda t))\right)(1)\right]
$$

with

$$
\mu_{0}=\left(I_{0^{+}}^{\alpha-1}(\exp (-\lambda t))\right)(1)-\left(I_{0^{+}}^{\alpha-1+\gamma}(\exp (-\lambda t))\right)(1) .
$$

We recall and summarize a useful result ([28]).

Lemma 8. Let E be a separable Banach space. Let $G$ be the function defined by (11)-(13).

(i) $G(\cdot, \cdot)$ satisfies the following estimate

$$
|G(t, s)| \leq \frac{1}{\Gamma(\alpha)}\left(\frac{1+\Gamma(\gamma+1)}{\left|\mu_{0}\right| \Gamma(\alpha) \Gamma(\gamma+1)}+1\right)=M_{G} .
$$

(ii) If $u \in W_{B, E}^{\alpha, 1}([0,1])$ satisfying boundary conditions (7), then

$$
u(t)=\int_{0}^{1} G(t, s)\left(D^{\alpha} u(s)+\lambda D^{\alpha-1} u(s)\right) d s \quad \text { for every } t \in[0,1] .
$$

(iii) Let $f \in L_{E}^{1}([0,1])$ and let $u_{f}:[0,1] \rightarrow E$ be the function defined by

$$
u_{f}(t):=\int_{0}^{1} G(t, s) f(s) d s \quad \text { for } \quad t \in[0,1]
$$

Then,

$$
\left.I_{0^{+}}^{\beta} u_{f}(t)\right|_{t=0}=0 \quad \text { and } \quad u_{f}(1)=\left(I_{0^{+}}^{\gamma} u_{f}\right)(1) .
$$

Moreover $u_{f} \in W_{B, E}^{\alpha, 1}([0,1])$ and we have

$$
\begin{gathered}
\left(D^{\alpha-1} u_{f}\right)(t)=\int_{0}^{t} \exp (-\lambda(t-s)) f(s) d s+\exp (-\lambda t) \int_{0}^{1} \varphi(s) f(s) d s \quad \text { for } t \in[0,1] \\
\left(D^{\alpha} u_{f}\right)(t)+\lambda\left(D^{\alpha-1} u_{f}\right)(t)=f(t) \quad \text { for all } t \in[0,1]
\end{gathered}
$$

Remark 2. From Lemma 8, we can claim that, if

$$
u_{f}(t)=\int_{0}^{1} G(t, s) f(s) d s, \quad f \in L_{E}^{1}([0,1]),
$$

then, for all $t \in[0,1]$,

$$
\left\|u_{f}(t)\right\| \leq M_{G}\|f\|_{L_{E}^{1}([0,1])} \text { and }\left\|D^{\alpha-1} u_{f}(t)\right\| \leq M_{G}\|f\|_{L_{E}^{1}([0,1])},
$$

Indeed, by Lemma 8(i), it suffices to prove that $\left\|D^{\alpha-1} u_{f}(t)\right\| \leq M_{G}\|f\|_{L_{E}^{1}([0,1])}$. It follows from (14) that

$$
\left\|D^{\alpha-1} u_{f}(t)\right\| \leq \int_{0}^{1}(1+|\varphi(s)|)|f(s)| d s .
$$

This, by an increase of $\varphi$ (See [28] (2.9)), gives

$$
\left\|D^{\alpha-1} u_{f}(t)\right\| \leq \Gamma(\alpha) M_{G}\|f\|_{L_{E}^{1}([0,1])}
$$


and, since $\alpha \in[1,2]$, implies our conclusion.

\subsection{Topological Structure of the Solution Set}

From Lemma 8, we summarize a crucial fact.

Lemma 9. Let $E$ be a separable Banach space. Let $f \in L^{1}(I, E, d t)$. Then, the boundary value problem

$$
\left\{\begin{array}{l}
D^{\alpha} u(t)+\lambda D^{\alpha-1} u(t)=f(t), \quad t \in I \\
\left.I_{0^{+}}^{\beta} u(t)\right|_{t=0}=0, \quad u(1)=I_{0^{+}}^{\gamma} u(1)
\end{array}\right.
$$

has a unique $W_{B, E}^{\alpha, 1}(I)$-solution defined by

$$
u(t)=\int_{0}^{1} G(t, s) f(s) d s, t \in I .
$$

Theorem 3. Let $E$ be a separable Banach space. Let $X: I \rightarrow E$ be a convex compact valued measurable multifunction such that $X(t) \subset \gamma \bar{B}_{E}$ for all $t \in I$, where $\gamma$ is a positive constant and $S_{X}^{1}$ be the set of all measurable selections of $X$. Then, the $W_{B, E}^{\alpha, 1}(I)$-solutions set of problem

$$
\left\{\begin{array}{l}
D^{\alpha} u(t)+\lambda D^{\alpha-1} u(t)=f(t), f \in S_{X^{1}}^{1}, \text { a.e. } t \in I \\
\left.I_{0^{+}}^{\beta} u(t)\right|_{t=0}=0, \quad u(1)=I_{0^{+}}^{\gamma} u(1)
\end{array}\right.
$$

is compact in $C_{E}(I)$.

Proof. By virtue of Lemma 6 , the $W_{B, E}^{\alpha, 1}([0,1])$-solutions set $\mathcal{X}$ to the above inclusion is characterized by

$$
\mathcal{X}=\left\{u_{f}: I \rightarrow E, u_{f}(t)=\int_{0}^{1} G(t, s) f(s) d s, f \in S_{X}^{1}, t \in I\right\}
$$

Claim: $\mathcal{X}$ is bounded, convex, equicontinuous and compact in $C_{E}(I)$.

From definition of the Green function $G$, it is not difficult to show that $\left\{u_{f}: f \in S_{X}^{1}\right\}$ is bounded, equicontinuous in $C_{E}(I)$. Indeed, let $\left(u_{f_{n}}\right)$ be a sequence in $\mathcal{X}$. We note that, for each $n \in \mathbb{N}$, we have $u_{f_{n}} \in W_{B, E}^{\alpha, 1}(I)$, and

$$
u_{f_{n}}(t)=\int_{0}^{1} G(t, s) f_{n}(s) d s, \quad t \in I,
$$

with

- $\left.\quad I_{0^{+}}^{\beta} u_{f_{n}}(t)\right|_{t=0}=0, u_{f_{n}}(1)=I_{0^{+}}^{\gamma} u(1)$,

- $\quad\left(D^{\alpha-1} u_{f_{n}}\right)(t)=\int_{0}^{t} \exp (-\lambda(t-s)) f_{n}(s) d s+\exp (-\lambda t) \int_{0}^{1} \varphi(s) f_{n}(s) d s, \quad t \in I$,

- $\quad\left(D^{\alpha} u_{f_{n}}\right)(t)+\lambda\left(D^{\alpha-1} u_{f_{n}}\right)(t)=f_{n}(t), t \in I$. 
For $t_{1}, t_{2} \in I, t_{1}<t_{2}$, we have

$$
\begin{aligned}
& u_{f_{n}}\left(t_{2}\right)-u_{f_{n}}\left(t_{1}\right)=\int_{0}^{1} G(t, s)\left(f_{n}\left(t_{2}, s\right)-f_{n}\left(t_{1}, s\right)\right) d s \\
& =\int_{0}^{1} \varphi(s) f_{n}(s) d s\left(\int_{0}^{t_{2}} \frac{e^{-\lambda \tau}}{\Gamma(\alpha-1)}\left(t_{2}-\tau\right)^{\alpha-2} d \tau-\int_{0}^{t_{1}} \frac{e^{-\lambda \tau}}{\Gamma(\alpha-1)}\left(t_{1}-\tau\right)^{\alpha-2} d \tau\right) \\
& +\int_{0}^{t_{2}} e^{\lambda s}\left(\int_{s}^{t_{2}} \frac{\left(t_{2}-\tau\right)^{\alpha-2}}{\Gamma(\alpha-1)} e^{-\lambda \tau} d \tau\right) f(s) d s-\int_{0}^{t_{1}} e^{\lambda s}\left(\int_{s}^{t_{1}} \frac{e^{-\lambda \tau}}{\Gamma(\alpha-1)}\left(t_{1}-\tau\right)^{\alpha-2} d \tau\right) f(s) d s \\
& =\int_{0}^{1} \phi(s) f(s) d s\left[\int_{0}^{t_{1}} e^{-\lambda \tau} \frac{\left(t_{2}-\tau\right)^{\alpha-2}-\left(t_{1}-\tau\right)^{\alpha-2}}{\Gamma(\alpha-1)} d \tau+\int_{t_{1}}^{t_{2}} e^{-\lambda \tau} \frac{\left(t_{2}-\tau\right)^{\alpha-2}}{\Gamma(\alpha-1)} d \tau\right] \\
& \quad+\int_{0}^{t_{1}} e^{\lambda s}\left(\int_{s}^{t_{1}} e^{-\lambda \tau} \frac{\left(t_{2}-\tau\right)^{\alpha-2}-\left(t_{1}-\tau\right)^{\alpha-2}}{\Gamma(\alpha-1)} d \tau\right) f(s) d s \\
& \quad+\int_{0}^{t_{1}} e^{\lambda s}\left(\int_{t_{1}}^{t_{2}} e^{-\lambda \tau} \frac{\left(t_{2}-\tau\right)^{\alpha-2}}{\Gamma(\alpha-1)} d \tau\right) f(s) d s+\int_{t_{1}}^{t_{2}} e^{\lambda s}\left(\int_{s}^{t_{2}} \frac{\left(t_{2}-\tau\right)^{\alpha-2}}{\Gamma(\alpha-1)} e^{-\lambda \tau} d \tau\right) f(s) d s .
\end{aligned}
$$

Then, we get

$$
\begin{aligned}
\left\|u_{f_{n}}\left(t_{2}\right)-u_{f_{n}}\left(t_{1}\right)\right\| \leq & \int_{0}^{1}\left(|\varphi(s)|+e^{\lambda s}\right)|X(s)| d s \int_{0}^{t_{1}} e^{-\lambda \tau} \frac{\left(t_{1}-\tau\right)^{\alpha-2}-\left(t_{2}-\tau\right)^{\alpha-2}}{\Gamma(\alpha-1)} d \tau \\
& +\int_{0}^{1}\left(|\varphi(s)|+e^{\lambda s}\right)|X(s)| d s \int_{t_{1}}^{t_{2}} e^{-\lambda \tau} \frac{\left(t_{2}-\tau\right)^{\alpha-2}}{\Gamma(\alpha-1)} d \tau \\
& +\int_{t_{1}}^{t_{2}} e^{\lambda s}|X(s)| d s \int_{t_{1}}^{t_{2}} e^{-\lambda \tau} \frac{\left(t_{2}-\tau\right)^{\alpha-2}}{\Gamma(\alpha-1)} d \tau .
\end{aligned}
$$

It is easy to obtain, after an integration by part, that

$$
\int_{t_{1}}^{t_{2}} e^{-\lambda \tau} \frac{\left(t_{2}-\tau\right)^{\alpha-2}}{\Gamma(\alpha-1)} d \tau=e^{-\lambda t_{1}} \frac{\left(t_{2}-t_{1}\right)^{\alpha-2}}{\Gamma(\alpha)}+\lambda \int_{t_{1}}^{t_{2}} e^{-\lambda \tau} \frac{\left(t_{2}-\tau\right)^{\alpha-1}}{\Gamma(\alpha)} d \tau \leq \frac{1+\lambda}{\Gamma(\alpha)}\left(t_{2}-t_{1}\right)^{\alpha-1}
$$

and

$$
\begin{gathered}
\int_{0}^{t_{1}} e^{-\lambda \tau} \frac{\left(t_{1}-\tau\right)^{\alpha-2}-\left(t_{2}-\tau\right)^{\alpha-2}}{\Gamma(\alpha-1)} d \tau \leq \int_{0}^{t_{1}} \frac{\left(t_{1}-\tau\right)^{\alpha-2}-\left(t_{2}-\tau\right)^{\alpha-2}}{\Gamma(\alpha-1)} d \tau \\
=\frac{\left(t_{2}-t_{1}\right)^{\alpha-1}+t_{1}^{\alpha-1}-t_{2}^{\alpha-1}}{\Gamma(\alpha)}
\end{gathered}
$$

Using the inequality that $\left|a^{p}-b^{p}\right| \leq|a-b|^{p}$ for all $a, b \geq 0$ and $0<p \leq 1$, we yield

$$
\int_{0}^{t_{1}} e^{-\lambda \tau} \frac{\left(t_{2}-\tau\right)^{\alpha-2}-\left(t_{1}-\tau\right)^{\alpha-2}}{\Gamma(\alpha-1)} d \tau \leq \frac{2}{\Gamma(\alpha)}\left(t_{2}-t_{1}\right)^{\alpha-1}
$$

Then, since $\alpha \in] 1,2]$, we can increase $\left\|u_{f_{n}}\left(t_{2}\right)-u_{f_{n}}\left(t_{1}\right)\right\|$ by

$$
\left\|u_{f_{n}}\left(t_{2}\right)-u_{f_{n}}\left(t_{1}\right)\right\| \leq K\left|t_{2}-t_{1}\right|^{\alpha-1}
$$

with $K=\int_{0}^{1}\left[(3+\lambda)|\phi(s)|+(4+2 \lambda) e^{\lambda s}\right]|X(s)| d s$ This shows that $\left\{u_{f_{n}}: n \in \mathbf{N}\right\}$ is equicontinuous in $C_{E}(I)$. Moreover, for each $t \in I$, the set $\left\{u_{f_{n}}(t): n \in \mathbf{N}\right\}$ is contained in the convex compact set $\int_{0}^{1} G(t, s) X(s) d s[27,29]$ so that $\mathcal{X}$ is relatively compact in $C_{E}(I)$ as claimed. Thus, we can assume that

$$
\lim _{n \rightarrow \infty} u_{f_{n}}=u_{\infty} \in C_{E}(I)
$$


As $S_{X}^{1}$ is $\sigma\left(L_{E}^{1}, L_{E^{*}}^{\infty}\right)$-compact, e.g., [29], we may assume that $\left(f_{n}\right) \sigma\left(L_{E^{\prime}}^{1}, L_{E^{*}}^{\infty}\right)$-converges to $f_{\infty} \in S_{X^{\prime}}^{1}$ so that $u_{f_{n}}$ weakly converges to $u_{f_{\infty}}$ in $C_{E}(I)$ where $u_{f_{\infty}}(t)=\int_{0}^{1} G(t, s) f_{\infty}(s) d s$ and so, for every $t \in I$,

$$
u_{\infty}(t)=w-\lim _{n \rightarrow \infty} u_{f_{n}}(t)=w-\lim _{n \rightarrow \infty} \int_{0}^{1} G(t, s) f_{n}(s) d s=\int_{0}^{1} G(t, s) f_{\infty}(s) d s=u_{f_{\infty}}(t)
$$

and

$$
\begin{aligned}
w-\lim _{n \rightarrow \infty}\left(D^{\alpha-1} u_{f_{n}}\right)(t) & =w-\lim _{n \rightarrow \infty}\left[\int_{0}^{t} \exp (-\lambda(t-s)) f_{n}(s) d s+\exp (-\lambda t) \int_{0}^{1} \varphi(s) f_{n}(s) d s\right] \\
& =\int_{0}^{t} \exp (-\lambda(t-s)) f_{\infty}(s) d s+\exp (-\lambda t) \int_{0}^{1} \varphi(s) f_{\infty}(s) d s \\
& =\left(D^{\alpha-1} u_{f_{\infty}}\right)(t), \quad t \in I .
\end{aligned}
$$

This means $u_{\infty} \in \mathcal{X}$, and the proof of the theorem is complete.

Remark 3. In the course of the proof of Theorem 3, we have proven the continuous dependence of the mappings $f \mapsto u_{f}$ and $f \mapsto D^{\alpha-1} u_{f}$ on the convex $\sigma\left(L_{E}^{1}, L_{E^{*}}^{\infty}\right)$-compact set $S_{X}^{1}$. This fact has some importance in further applications.

Theorem 4. Let $I=[0,1]$. Let $(t, x) \rightarrow A_{(t, x)}: D\left(A_{(t, x)}\right) \rightarrow 2^{E}$ a maximal monotone operator satisfying: $\left(H_{1}\right)\left\|A_{(t, x)}^{0} y\right\| \leq c(1+\|x\|+\|y\|)$ for all $(t, x, y) \in I \times E \times D\left(A_{(t, x)}\right)$, for some positive constant $c$, $\left(H_{2}\right) \operatorname{dis}\left(A_{(t, x)}, A_{(\tau, y)}\right) \leq a(t)-a(\tau)+r\|x-y\|$, for all $0 \leq \tau \leq t \leq 1$ and for all $(x, y) \in E \times E$, where $r$ is a positive number, $a: I \rightarrow\left[0,+\infty\left[\right.\right.$ is nondecreasing absolutely continuous on $I$ with $\dot{a} \in L^{2}(I, \mathbb{R}, d t)$,

$\left(H_{3}\right) D\left(A_{(t, x)}\right) \subset X(t) \subset \gamma \bar{B}_{E}$ for all $(t, x) \in I \times E$, where $X: I \rightarrow E$ is a convex compact valued measurable mapping and $\gamma$ is a positive number.

Then, there is a $W_{B, E}^{\alpha, 1}(I)$ mapping $x: I \rightarrow E$ and an absolutely continuous mapping $u: I \rightarrow E$ satisfying

$$
\left\{\begin{array}{l}
D^{\alpha} x(t)+\lambda D^{\alpha-1} x(t)=u(t), \quad t \in I \\
\left.I_{0^{+}}^{\beta} x(t)\right|_{t=0}=0, \quad x(1)=I_{0^{+}}^{\gamma} x(1) \\
u(t) \in D\left(A_{(t, x(t))}\right) \\
-\frac{d u}{d t}(t) \in A_{(t, x(t))} u(t) \quad \text { a.e. } t \in I .
\end{array}\right.
$$

Proof. Let us consider the convex compact subset $\mathcal{X}$ in the Banach space $\mathcal{C}_{E}(I)$ defined by

$$
\mathcal{X}:=\left\{u_{f}: I \rightarrow E: u_{f}(t)=\int_{0}^{1} G(t, s) f(s) d s, f \in S_{X}^{1}, t \in I\right\}
$$

We note that $\mathcal{X}$ is convex compact and equi-Lipschitz. Cf the proof of Theorem 3. Now, for each $h \in \mathcal{X}$, let us consider the unique absolutely continuous solution $u_{h}$ to

$$
\left\{\begin{array}{l}
-\dot{u}_{h}(t) \in A_{(t, h(t))} u_{h}(t) \quad \text { a.e. } t \in I \\
u_{h}(t) \in D\left(A_{(t, h(t))}\right), \forall t \in I \\
u_{h}(0)=u_{0} \in D\left(A_{(0, h(0))}\right)
\end{array}\right.
$$

For each $h$, let us set

$$
\Phi(h)(t)=\int_{0}^{1} G(t, s) u_{h}(s) d s, t \in I
$$


Since $u_{h}(s) \in D\left(A_{(s, h(s))}\right) \subset X(s)$, then it is clear that $\Phi(h) \in \mathcal{X}$.

Now, we check that $\Phi$ is continuous. It is sufficient to show that, if $\left(h_{n}\right)$ converges uniformly to $h$ in $\mathcal{X}$, then the absolutely continuous solution $u_{h_{n}}$ associated with $h_{n}$

$$
\left\{\begin{array}{r}
u_{h_{n}}(0)=u_{0}^{n} \in D\left(A_{\left(0, h_{n}(0)\right)}\right) \\
u_{h_{n}}(t) \in D\left(A_{\left(t, h_{n}(t)\right)}\right), \forall t \in I \\
-\dot{u}_{h_{n}}(t) \in A_{\left(t, h_{n}(t)\right)} u_{h_{n}}(t) \quad \text { a.e. } t \in I
\end{array}\right.
$$

uniformly converges to the absolutely solution $u_{h}$ associated with $h$

$$
\left\{\begin{array}{r}
u_{h}(0)=u_{0} \in D\left(A_{(0, h(0))}\right) \\
u_{h}(t) \in D\left(A_{(t, h(t))}\right), \forall t \in[0, T] \\
-\dot{u}_{h}(t) \in A_{(t, h(t))} u_{h}(t) \quad \text { a.e. } t \in[0, T]
\end{array}\right.
$$

This fact is ensured by repeating the proof of Theorem 1 . Since $h_{n} \rightarrow h$, we have

$$
\begin{gathered}
\Phi\left(h_{n}\right)(t)-\Phi(h)(t)=\int_{0}^{1} G(t, s) u_{h_{n}}(s) d s-\int_{0}^{1} G(t, s) u_{h}(s) d s \\
=\int_{0}^{1} G(t, s)\left[u_{h_{n}}(s)-u_{h}(s)\right] d s \\
\leq \int_{0}^{1} M_{G}\left\|u_{h_{n}}(s)-u_{h}(s)\right\| d s
\end{gathered}
$$

As $\left\|u_{h_{n}}(\cdot)-u_{h}(\cdot)\right\| \rightarrow 0$ uniformly, we conclude that

$$
\sup _{t \in I}\left\|\Phi\left(h_{n}\right)(t)-\Phi(h)(t)\right\| \leq \int_{0}^{1} M_{G}\left\|u_{h_{n}}(\cdot)-u_{h}(\cdot)\right\| d s \rightarrow 0
$$

so that $\Phi\left(h_{h}\right) \rightarrow \Phi(h)$ in $\mathcal{C}_{E}(I)$. Since $\Phi: \mathcal{X} \rightarrow \mathcal{X}$ is continuous, $\Phi$ has a fixed point, say $h=\Phi(h) \in \mathcal{X}$. This means that

$$
h(t)=\Phi(h)(t)=\int_{0}^{1} G(t, s) u_{h}(s) d s,
$$

with

$$
\left\{\begin{array}{r}
u_{h}(0) \in D\left(A_{(0, h(0))}\right) \\
u_{h}(t) \in D\left(A_{(t, h(t))}\right), \forall t \in I \\
-\dot{u}_{h}(t) \in A_{(t, h(t))} u_{h}(t) \quad \text { a.e. } t \in I
\end{array}\right.
$$

Coming back to Lemma 9 and applying the above notations, this means that we have just shown that there exists a mapping $h \in W_{E}^{\alpha, \infty}(I)$ satisfying

$$
\left\{\begin{array}{r}
D^{\alpha} h(t)+\lambda D^{\alpha-1} h(t)=u_{h}(t), \\
\left.I_{0^{+}}^{\beta} h(t)\right|_{t=0}=0, \quad h(1)=I_{0^{+}}^{\gamma} h(1) \\
u_{h}(0) \in D\left(A_{(0, h(0))}\right) \\
u_{h}(t) \in D\left(A_{(t, h(t))}\right), \forall t \in I \\
-\dot{u}_{h}(t) \in A_{(t, h(t))} u_{h}(t) \quad \text { a.e. } t \in I
\end{array}\right.
$$

Now, we present an extension of the preceding theorem dealing with a Lipschitz perturbation. 
Theorem 5. Let $I=[0,1]$. Let $(t, x) \rightarrow A_{(t, x)}: D\left(A_{(t, x)}\right) \rightarrow 2^{E}$ a maximal monotone operator satisfying: $\left(H_{1}\right)\left\|A_{(t, x)}^{0} y\right\| \leq c(1+\|x\|+\|y\|)$ for all $(t, x, y) \in I \times E \times D\left(A_{(t, x)}\right)$, for some positive constant $c$, $\left(H_{2}\right) \operatorname{dis}\left(A_{(t, x)}, A_{(\tau, y)}\right) \leq a(t)-a(\tau)+r\|x-y\|$, for all $0 \leq \tau \leq t \leq 1$ and for all $(x, y) \in E \times E$, where $r$ is a positive number, $a: I \rightarrow\left[0,+\infty\left[\right.\right.$ is nondecreasing absolutely continuous on $I$ with $\dot{a} \in L^{2}(I, \mathbb{R}, d t)$,

$\left(H_{3}\right) D\left(A_{(t, x)}\right) \subset X(t) \subset \gamma \bar{B}_{E}$ for all $(t, x) \in I \times E$, where $X: I \rightarrow E$ is a convex compact valued measurable mapping and $\gamma$ is a positive number.

Let $f: I \times E \times E \rightarrow E$ such that

(i) $f(., x, y)$ is Lebesgue measurable on I for all $(x, y) \in E \times E$

(ii) $f(t, . .$.$) is continuous on E \times E$,

(iii) $\|f(t, x, y)\| \leq M$ for all $(t, x, y) \in I \times E \times E$,

(iv) $\|f(t, x, y)-f(t, x, z)\| \leq M\|y-z\|$, for all $(t, x, y, z) \in I \times E \times E \times E$

for some positive constant $M$.

Then, there is a $W_{B, E}^{\alpha, 1}(I)$ mapping $x: I \rightarrow E$ and an absolutely continuous mapping $v: I \rightarrow E$ satisfying

$$
\left\{\begin{array}{l}
D^{\alpha} x(t)+\lambda D^{\alpha-1} x(t)=v(t), t \in I \\
\left.I_{0^{+}}^{\beta} x(t)\right|_{t=0}=0, \quad x(1)=I_{0^{+}}^{\gamma} x(1) \\
v(t) \in D\left(A_{(t, x(t))}\right), t \in I \\
-\frac{d v}{d t}(t) \in A_{(t, x(t))} v(t)+f(t, x(t), v(t)) \quad \text { a.e. } t \in I .
\end{array}\right.
$$

Proof. Let us consider the convex compact subset $\mathcal{X}$ in the Banach space $\mathcal{C}_{E}(I)$ defined by

$$
\mathcal{X}:=\left\{u_{f}: I \rightarrow E: u_{f}(t)=\int_{0}^{1} G(t, s) f(s) d s, f \in S_{X}^{1}, t \in I\right\}
$$

We note that $\mathcal{X}$ is convex compact and equi-Lipschitz. Cf the proof of Theorem 3 . Now, for each $h \in \mathcal{X}$, let us consider the unique absolutely continuous solution $u_{h}$ to

$$
\left\{\begin{array}{l}
-\dot{u}_{h}(t) \in A_{(t, h(t))} u_{h}(t)+f\left(t, h(t), u_{h}(t)\right) \quad \text { a.e. } t \in I \\
u_{h}(t) \in D\left(A_{(t, h(t))}\right), \forall t \in I \\
u_{h}(0)=u_{0} \in D\left(A_{(0, h(0))}\right)
\end{array}\right.
$$

Existence and uniqueness of absolutely solution $u_{h}$ are ensured by the fact that the operator $B_{h}(t)=A_{(t, h(t))}$ is a time dependent maximal monotone operator absolutely continuous in variation (See Lemma 5), and the mapping $f_{h}(t, x):=f(t, h(t), y)$ is measurable with $t \in I$ and Lipschitz with $y \in E$. Furthermore, we have the estimate $\left\|\dot{u}_{h}(t)\right\| \leq \psi(t)$ a.e for all $h \in \mathcal{X}$ where $\psi \in L^{2}(I)$ by the consideration given in Lemma 5 and the estimate of velocity given in ([22], Theorem 1). For each $h$, let us set

$$
\Phi(h)(t)=\int_{0}^{1} G(t, s) u_{h}(s) d s, t \in I .
$$

Since $u_{h}(s) \in D\left(A_{(s, h(s))}\right) \subset X(s)$, then it is clear that $\Phi(h) \in \mathcal{X}$.

Now, we check that $\Phi$ is continuous. It is sufficient to show that, if $\left(h_{n}\right)$ converges uniformly to $h$ in $\mathcal{X}$, then the absolutely continuous solution $u_{h_{n}}$ associated with $h_{n}$

$$
\left\{\begin{array}{r}
u_{h_{n}}(0)=u_{0}^{n} \in D\left(A_{\left(0, h_{n}(0)\right)}\right) \\
u_{h_{n}}(t) \in D\left(A_{\left(t, h_{n}(t)\right)}\right), \forall t \in I \\
-\dot{u}_{h_{n}}(t) \in A_{\left(t, h_{n}(t)\right)} u_{h_{n}}(t)+f\left(t, h_{n}(t), u_{h_{n}}(t)\right) \text { a.e. } t \in I
\end{array}\right.
$$


uniformly converges to the absolutely solution $u_{h}$ associated with $h$

$$
\left\{\begin{array}{r}
u_{h}(0) \in D\left(A_{(0, h(0))}\right) \\
u_{h}(t) \in D\left(A_{(t, h(t))}\right), \forall t \in I \\
-\dot{u}_{h}(t) \in A_{(t, h(t))} u_{h}(t)+f\left(t, h(t), u_{h}(t)\right) \text { a.e. } t \in I
\end{array}\right.
$$

This need careful look. We note that $u_{h_{n}}$ is equicontinuous with $\left\|\dot{u}_{h_{n}}(t)\right\| \leq \psi(t)$ for almost all $t \in I$ and for all $n \in N$ where $\psi \in L^{2}$ and $u_{h_{n}}(t) \in D\left(A_{\left(t, h_{n}(t)\right)}\right) \subset X(t)$ for all $t \in I$ and for all $n \in N$. Thus, by extracting subsequence, we may assume that $u_{h_{n}}(t) \rightarrow v(t)=v(0)+\int_{0}^{t} \dot{v}(s) d s$ with $\dot{v} \in L_{E}^{2}(I)$ for all $t \in I$ and $\dot{u}_{h_{n}} \rightarrow \dot{v}$ weakly in $L_{E}^{2}(I)$. Let us check that $v(t) \in D\left(A_{(t, h(t))}\right)$ for all $t \in I$. We have $\operatorname{dis}\left(A_{\left(t, h_{n}(t)\right.}, A_{(t, h(t))}\right) \leq r\left\|h_{n}(t)-h(t)\right\| \rightarrow 0$. It is clear that $\left(y_{n}=A_{\left(t, h_{n}(t)\right.}^{0} u_{h_{n}}(t)\right)$ is bounded and hence relatively weakly compact. By applying Lemma 2 to $u_{h_{n}}(t) \rightarrow v(t)$ and to a convergence subsequence of $\left(y_{n}\right)$ using $u_{h_{n}}(t) \in X(t) \subset \gamma \bar{B}_{E}$ to show that $v(t) \in D\left(A_{(t, h(t))}\right)$. As $\dot{u}_{h_{n}} \rightarrow \dot{v}$ weakly in $L_{E}^{2}(I), \dot{u}_{h_{n}} \rightarrow \dot{v}$ Komlos. Note that $f\left(t, h_{n}(t), u_{h_{n}}(t)\right) \rightarrow f\left(t, h(t), u_{h}(t)\right)$ weakly in $L_{E}^{2}(I)$. Thus, $z_{n}(t):=f\left(t, h_{n}(t), u_{h_{n}}(t)\right) \rightarrow z(t):=f(t, h(t), v(t))$ Komlos. Hence, $\dot{u}_{h_{n}}(t)+f\left(t, h_{n}(t), u_{h_{n}}(t) \rightarrow\right.$ $\dot{v}(t)+f(t, h(t), v(t))$ Komlos. Apply Lemma 4 to $A_{\left(t, h_{n}(t)\right)}$ and $A_{(t, h(t))}$ to find a sequence $\left(\eta_{n}\right)$ such that such that $\eta_{n} \in D\left(A_{\left(t, h_{n}(t)\right)}\right), \eta_{n} \rightarrow \eta, A_{\left(t, h_{n}(t)\right.}^{0} \eta_{n} \rightarrow A_{(t, h(t))}^{0} v(t)$ From

$$
-\dot{u}_{h_{n}}(t) \in A_{\left(t, h_{n}(t)\right)} u_{h_{n}}(t)+f\left(t, h_{n}(t), u_{h_{n}}(t)\right)(* *)
$$

by monotonicity

$$
\left.\left.\left\langle\frac{d u_{h_{n}}}{d t}+z_{n}(t), u_{h_{n}}(t)-\eta_{n}\right\rangle \leq A_{\left(t, h_{n}(t)\right)}^{0} \eta_{n}, \eta_{n}-u_{h_{n}}(t)\right\rangle \cdot .^{* * *}\right)
$$

From

$$
\begin{gathered}
\left\langle\frac{d u_{h_{n}}}{d t}(t)+z_{n}(t), v(t)-\eta\right\rangle=\left\langle\frac{d u_{h_{n}}}{d t}(t)+z_{n}(t), u_{h_{n}}(t)-\eta_{n}\right\rangle \\
+\left\langle\frac{d u_{h_{n}}}{d t}(t)+z_{n}(t), v(t)-u_{h_{n}}(t)-\left(\eta-\eta_{n}\right)\right\rangle,
\end{gathered}
$$

let us write

$$
\begin{aligned}
& \frac{1}{n} \sum_{j=1}^{n}\left\langle\frac{d u_{h_{j}}}{d t}(t)+z_{j}(t), v(t)-\eta\right\rangle= \\
& \frac{1}{n} \sum_{j=1}^{n}\left\langle\frac{d u_{h_{j}}}{d t}(t)+z_{j}(t), u_{h_{j}}(t)-\eta_{j}\right\rangle+\frac{1}{n} \sum_{j=1}^{n}\left\langle\frac{d u_{h_{j}}}{d t}(t)+z_{j}(t), v(t)-u_{h_{j}}(t)\right\rangle \\
&+\sum_{j=1}^{n}\left\langle\frac{d u_{h_{j}}}{d t}(t)+z_{j}(t), \eta_{j}-\eta\right\rangle,
\end{aligned}
$$

so that

$$
\begin{aligned}
\frac{1}{n} \sum_{j=1}^{n}\left\langle\frac{d u_{h_{j}}}{d t}(t)+z_{j}(t), v(t)-\eta\right\rangle \leq & \left.\frac{1}{n} \sum_{j=1}^{n}\left\langle A_{\left(t, h_{j}(t)\right)}^{0} \eta_{j}, \eta_{j}-u_{h_{j}}(t)\right\rangle+(\psi(t)+M) \frac{1}{n} \sum_{j=1}^{n} \| v(t)-u_{h_{j}}(t)\right) \| . \\
& +(\psi(t)+M) \frac{1}{n} \sum_{j=1}^{n}\left\|\eta_{j}-\eta\right\| .
\end{aligned}
$$

Passing to the limit using (5) when $n \rightarrow \infty$, this last inequality gives immediately

$$
\left\langle\frac{d v}{d t}(t)+z(t), v(t)-\eta\right\rangle \leq\left\langle A_{(t, h(t))}^{0} \eta, \eta-v(t)\right\rangle \text { a.e. }
$$


As a consequence, by Lemma 1, we get

$-\frac{d v}{d t}(t) \in A_{(t, h(t))} v(t)+z(t)$ a.e. with $v(t) \in D\left(A_{(t, h(t))}\right)$ for all $t \in I$ so that, by uniqueness, $v=u_{h}$. Since $h_{n} \rightarrow h$, we have

$$
\begin{gathered}
\Phi\left(h_{n}\right)(t)-\Phi(h)(t)=\int_{0}^{1} G(t, s) u_{h_{n}}(s) d s-\int_{0}^{1} G(t, s) u_{h}(s) d s \\
=\int_{0}^{1} G(t, s)\left[u_{h_{n}}(s)-u_{h}(s)\right] d s \\
\leq \int_{0}^{1} M_{G}\left\|u_{h_{n}}(s)-u_{h}(s)\right\| d s
\end{gathered}
$$

As $\left\|u_{h_{n}}(\cdot)-u_{h}(\cdot)\right\| \rightarrow 0$ uniformly, we conclude that

$$
\sup _{t \in I}\left\|\Phi\left(h_{n}\right)(t)-\Phi(h)(t)\right\| \leq \int_{0}^{1} M_{G}\left\|u_{h_{n}}(\cdot)-u_{h}(\cdot)\right\| d s \rightarrow 0
$$

so that $\Phi\left(h_{n}\right) \rightarrow \Phi(h)$ in $\mathcal{C}_{E}(I)$. Since $\Phi: \mathcal{X} \rightarrow \mathcal{X}$ is continuous, $\Phi$ has a fixed point, say $h=\Phi(h) \in \mathcal{X}$ This means that

$$
h(t)=\Phi(h)(t)=\int_{0}^{1} G(t, s) u_{h}(s) d s,
$$

with

$$
\left\{\begin{array}{r}
u_{h}(0) \in D\left(A_{(0, h(0))}\right) \\
u_{h}(t) \in D\left(A_{(t, h(t))}\right), \forall t \in I \\
-\dot{u}_{h}(t) \in A_{(t, h(t))} u_{h}(t)+f\left(t, h(t), u_{h}(t)\right) \text { a.e. } t \in I
\end{array}\right.
$$

Coming back to Lemma 9 and applying the above notations, this means that we have just shown that there exists a mapping $h \in W_{B, E}^{\alpha, \infty}(I)$ satisfying

$$
\left\{\begin{array}{r}
D^{\alpha} h(t)+\lambda D^{\alpha-1} h(t)=u_{h}(t), \\
\left.I_{0^{+}}^{\beta} h(t)\right|_{t=0}=0, \quad h(1)=I_{0^{+}}^{\gamma} h(1) \\
u_{h}(0) \in D\left(A_{(0, h(0))}\right) \\
u_{h}(t) \in D\left(A_{(t, h(t))}\right), \forall t \in I \\
-\dot{u}_{h}(t) \in A_{(t, h(t))} u_{h}(t)+f\left(t, h(t), u_{h}(t)\right) \text { a.e. } t \in I
\end{array}\right.
$$

We finish the paper by investigating a fractional order to a sweeping process [30,31].

We begin recall the existence of absolutely continuous solution to a class of sweeping process [18,32].

Theorem 6. Let $f:[0, T] \rightarrow E$ be a continuous mapping such that $\|f(t)\| \leq \beta$ for all $t \in[0, T]$, let $v$ : $[0, T] \rightarrow \mathbf{R}^{+}$be a positive nondecreasing continuous function with $v(0)=0$. Let $C:[0, T] \rightarrow E$ be a convex weakly compact valued mapping such that $d_{H}(C(t), C(\tau)) \leq|v(t)-v(\tau)|$ for all $t, \tau \in[0, T]$. Let $A: E \rightarrow E$ be a linear continuous coercive symmetric operator and let $B: E \rightarrow E$ be a linear continuous compact operator. Then, for any $u_{0} \in E$, the evolution inclusion

$$
\begin{gathered}
f(t)+B u(t)-A \frac{d u}{d t}(t) \in N_{C(t)}\left(\frac{d u}{d t}(t)\right) \\
u(0)=u_{0}
\end{gathered}
$$

admits a unique $W_{E}^{1, \infty}([0, T])$ solution $u:[0, T] \rightarrow E$. 
Theorem 7. Let $f: I \times E \rightarrow E$ be a bounded continuous mapping such that $\|f(t, x)\| \leq M$ for all $(t, x) \in$ $I \times E$, for some positive constant $M$, let $v: I \rightarrow \mathbf{R}^{+}$be a positive nondecreasing continuous function with $v(0)=0$. Let $C: I \rightarrow$ E be a convex compact valued mapping such that $d_{H}(C(t), C(\tau)) \leq|v(t)-v(\tau)|$ for all $t, \tau \in I$. Let $A: E \rightarrow E$ be a linear continuous coercive symmetric operator and let $B: E \rightarrow E$ be a linear continuous compact operator.

Then, for any $u_{0} \in E$, there exists a $W_{B, E}^{\alpha, 1}(I)$ mapping $x: I \rightarrow E$ and an absolutely continuous mapping $u: I \rightarrow$ E satisfying

$$
\left\{\begin{array}{l}
u(0)=u_{0} \in E \\
D^{\alpha} x(t)+\lambda D^{\alpha-1} x(t)=u(t), t \in I \\
\left.I_{0^{+}}^{\beta} x(t)\right|_{t=0}=0, \quad x(1)=I_{0^{+}}^{\gamma} x(1) \\
\left.f(t, x(t))+B u(t)-A \frac{d u}{d t}(t)\right) \in N_{C(t)}\left(\frac{d u}{d t}(t)\right), \text { a.e. } t \in I
\end{array}\right.
$$

Proof. By Theorem 6 and the assumptions on $f$, for any bounded continuous mapping $h: I \rightarrow E$, there is a unique absolutely continuous solution $v_{h}$ to the inclusion

$$
\left\{\begin{array}{l}
v_{h}(0)=u_{0} \in E \\
\left.f(t, h(t))+B v_{h}(t)-A \frac{d v_{h}}{d t}(t)\right) \in N_{C(t)}\left(\frac{d v_{h}}{d t}(t)\right), \text { a.e. } t \in I
\end{array}\right.
$$

with $\frac{d v_{h}}{d t}(t) \in C(t)$ a.e. so that $v_{h}(t)=u_{0}+\int_{0}^{t} \frac{d v_{h}}{d s}(s) d s \in u_{0}+\int_{0}^{t} C(s) d s, \forall t \in I$. By our assumption, $C$ is scalarly upper semicontinuous convex compact valued integrably bounded: $C(t) \subset \rho \bar{B}_{E}, \forall t \in I$, hence, by [33], $t \mapsto \Psi(t):=u_{0}+\int_{0}^{t} C(s) d s$ is a scalarly upper semicontinuous convex compact valued integrably bounded mapping with $\Psi(t):=u_{0}+\int_{0}^{t} C(s) d s \subset u_{0}+\rho \bar{B}_{E}, \forall t \in I$. Let us consider the closed convex subset $\mathcal{X}$ in the Banach space $\mathcal{C}_{E}(I)$ defined by

$$
\mathcal{X}:=\left\{u_{f}: I \rightarrow E: u_{f}(t)=\int_{0}^{1} G(t, s) f(s) d s, f \in S_{u_{0}+\rho \bar{B}_{E^{\prime}}}^{1} t \in I\right\},
$$

where $S_{u_{0}+\rho \bar{B}_{E}}^{1}$ denotes the set of all integrable selections of the convex weakly compact valued constant multifunction $u_{0}+\rho \bar{B}_{E}$. Now, for each $h \in \mathcal{X}$, let us consider the mapping defined by

$$
\Phi(h)(t):=\int_{0}^{t} G(t, s) v_{h}(s) d s
$$

for $t \in I$. Then, it is clear that $\Phi(h) \in \mathcal{X}$. Since $u_{0}+\int_{0}^{t} C(s) d s$ is a convex compact, $\Phi(\mathcal{X})$ is equicontinuous and relatively compact in the Banach space $\mathcal{C}_{E}(I)$ by virtue of Theorem 3 using the compactness of $\Psi(t)$. Now, we check that $\Phi$ is continuous. It is sufficient to show that, if $\left(h_{n}\right)$ uniformly converges to $h$ in $\mathcal{X}$, then the absolutely continuous solution $v_{h_{n}}$ associated with $h_{n}$

$$
\left\{\begin{array}{l}
v_{h_{n}}(0)=u_{0} \in E \\
\left.f\left(t, h_{n}(t)\right)+B v_{h_{n}}(t)-A \frac{d v_{h_{n}}}{d t}(t)\right) \in N_{C(t)}\left(\frac{d v_{h_{n}}}{d t}(t)\right), \text { a.e. } t \in I
\end{array}\right.
$$

uniformly converges to the absolutely continuous solution $v_{h}$ associated with $h$

$$
\left\{\begin{array}{l}
v_{h}(0)=u_{0} \in E \\
\left.f(t, h(t))+B v_{h}(t)-A \frac{d v_{h}}{d t}(t)\right) \in N_{C(t)}\left(\frac{d v_{h}}{d t}(t)\right), \text { a.e. } t \in I
\end{array}\right.
$$

As $\left(v_{h_{n}}\right)$ is equi-absolutely continuous with $\left.v_{h_{n}} t\right) \in u_{0}+\int_{0}^{t} C(s) d s, \forall t \in I$, we may assume that $\left(v_{h_{n}}\right)$ uniformly converges to an absolutely continuous mapping $z$. 
Since $v_{h_{n}}(t)=u_{0}+\int_{[0, t]} \frac{d v_{h_{n}}}{d s}(s) d s, t \in I$ and $\frac{d v_{h_{n}}}{d s}(s) \in C(s)$, a.e. $s \in I$, we may assume that $\left(\frac{d v_{h_{n}}}{d t}\right)$ weakly converges in $L_{E}^{1}(I)$ to $w \in L_{E}^{1}(I)$ with $w(t) \in C(t), t \in I$ so that

$$
\lim _{n} v_{h_{n}}(t)=u_{0}+\int_{0}^{t} w(s) d s:=u(t), t \in I
$$

By identifying the limits, we get

$$
u(t)=z(t)=u_{0}+\int_{0}^{t} w(s) d s
$$

with $\dot{u}=w$. Therefore, by applying the arguments in the variational limit result in [34], we get

$$
\left.f(t, h(t))+B u(t)-A \frac{d u}{d t}(t)\right) \in N_{C(t)}\left(\frac{d u}{d t}(t)\right), \text { a.e. } t \in I
$$

with $u(0)=u_{0} \in E$, so that, by uniqueness, $u=v_{h}$. Since $h_{n} \rightarrow h$, we have

$$
\begin{gathered}
\Phi\left(h_{n}\right)(t)-\Phi(h)(t)=\int_{0}^{1} G(t, s) v_{h_{n}}(s) d s-\int_{0}^{1} G(t, s) v_{h}(s) d s \\
=\int_{0}^{1} G(t, s)\left[v_{h_{n}}(s)-v_{h}(s)\right] d s \\
\leq \int_{0}^{1} M_{G}\left\|v_{h_{n}}(s)-v_{h}(s)\right\| d s
\end{gathered}
$$

As $\left\|v_{h_{n}}(\cdot)-v_{h}(\cdot)\right\| \rightarrow 0$ uniformly, we conclude that

$$
\sup _{t \in I}\left\|\Phi\left(h_{n}\right)(t)-\Phi(h)(t)\right\| \leq \int_{0}^{1} M_{G}\left\|v_{h_{n}}(\cdot)-v_{h}(\cdot)\right\| d s \rightarrow 0
$$

so that $\Phi\left(h_{n}\right) \rightarrow \Phi(h)$ in $\mathcal{C}_{E}(I)$. Since $\Phi: \mathcal{X} \rightarrow \mathcal{X}$ is continuous and $\Phi(\mathcal{X})$ is relatively compact in $\mathcal{C}_{E}(I)$, by $[25,26] \Phi$ has a fixed point, say $h=\Phi(h) \in \mathcal{X}$. This means that

$$
h(t)=\Phi(h)(t)=\int_{0}^{1} G(t, s) v_{h}(s) d s,
$$

with

$$
\left\{\begin{array}{l}
v_{h}(0)=u_{0} \in E \\
D^{\alpha} h(t)+\lambda D^{\alpha-1} h(t)=v_{h}(t), t \in I \\
\left.I_{0^{+}}^{\beta} h(t)\right|_{t=0}=0, \quad h(1)=I_{0^{+}}^{\gamma} h(1) \\
\left.f(t, h(t))+B v_{h}(t)-A \frac{d v_{h}}{d t}(t)\right) \in N_{C(t)}\left(\frac{d v_{h}}{d t}(t)\right), \text { a.e. } t \in I
\end{array}\right.
$$

The proof is complete.

Theorem 8. Theorems 6 and 7 results are inspired by some ideas in [18]. At this point, some variants are available, mainly when the second member is a time dependent subdifferential operator [35], namely, for any $u_{0} \in E$, there exists a $W_{B, E}^{\alpha, 1}(I)$ mapping $x: I \rightarrow E$ and an absolutely continuous mapping $u: I \rightarrow E$ satisfying

$$
\left\{\begin{array}{l}
u(0)=u_{0} \in E \\
D^{\alpha} x(t)+\lambda D^{\alpha-1} x(t)=u(t), t \in I \\
\left.I_{0^{+}}^{\beta} x(t)\right|_{t=0}=0, \quad x(1)=I_{0^{+}}^{\gamma} x(1) \\
f(t, x(t))+B u(t)-A \frac{d u}{d t}(t) \in \partial \varphi\left(t, \frac{d u}{d t}(t)\right), \text { a.e. } t \in I
\end{array}\right.
$$




\section{On a Fillipov Theorem}

We end this section with a Fillipov theorem and a relaxation theorem for the fractional differential inclusion

$$
\left\{\begin{array}{l}
D^{\alpha} u(t)+\lambda D^{\alpha-1} u(t) \in F(t, u(t)) \text {, a.e. } t \in I \\
\left.I_{0^{+}}^{\beta} u(t)\right|_{t=0}=0, \quad u(1)=I_{0^{+}}^{\gamma} u(1)
\end{array}\right.
$$

where $F: I \times E \rightarrow E$ is a closed valued Lipschitz mapping w.r.t.o $x \in E$.

Theorem 9. Assume that $E$ is a separable Banach space. Let $F: I \times E \rightarrow E$ be a closed valued $\mathcal{L}(I) \otimes$ $\mathcal{B}(E)$-measurable mapping such that

$\left(\mathcal{H}_{1}\right): d_{H}(F(t, x), F(t, y)) \leq l(t)\|x-y\|$ for all $t, x, y$ where $\left.l \in L_{\mathbf{R}}^{1}(I)\right)$ such that $\rho:=M_{G}\|l\|_{L_{\mathbf{R}}^{1}(I)}<1$.

Assume further that

$\left(\mathcal{H}_{2}\right)$ : there exists $g \in L_{E}^{1}(I)$ such that $d\left(g(t), F\left(t, u_{g}(t)\right)\right)<\frac{l(t)}{\sum_{n=1}^{\infty} n \rho^{n-1}}$ where $u_{g}(t)=$ $\int_{0}^{1} G(t, s) g(s) d s, \forall t \in I$.

Then, the fractional differential inclusion

$$
\left\{\begin{array}{l}
D^{\alpha} u(t)+\lambda D^{\alpha-1} u(t) \in F(t, u(t)) \text {, a.e. } t \in I \\
\left.I_{0^{+}}^{\beta} u(t)\right|_{t=0}=0, \quad u(1)=I_{0^{+}}^{\gamma} u(1)
\end{array}\right.
$$

has at least a $W_{B, E}^{\alpha, 1}(I)$-solution $u: I \rightarrow E$.

Proof. We use the ideas in the proof of Theorem 4.3 in [36], Remark 2 and Lemma 9.

It is worth mentioning that the series $\Lambda:=\sum_{n=1}^{\infty} n \rho^{n-1}$ is convergent. Indeed, we have

$$
\lim _{n \rightarrow \infty} \frac{(n+1) \rho^{n}}{n \rho^{n-1}}=\lim _{n \rightarrow \infty} \frac{n+1}{n} \rho=\rho<1 .
$$

Thus, by d'Alembert's ratio test, the series $\sum_{n=1}^{\infty} n \rho^{n-1}$ is convergent

Step 1. We shall construct inductively sequence $\left\{f_{n}(\cdot)\right\}_{n=1}^{\infty}$ where $f_{1}=g$ such that the following conditions are fulfilled, for all $n \geq 1$,

$$
\begin{gathered}
f_{n} \in L_{E}^{1}(I) \quad \text { and } \quad f_{n+1}(t) \in F\left(t, u_{f_{n}}(t)\right), t \in I, \\
\left\|f_{n+1}(t)-f_{n}(t)\right\| \leq(n+1) \rho^{n-1} l(t) \Lambda^{-1}, \\
\left\|u_{f_{n+1}}(t)-u_{f_{n}}(t)\right\|=\left\|\int_{0}^{1} G(t, s)\left[f_{n+1}(s)-f_{n}(s)\right] d s\right\| \leq(n+1) \rho^{n} \Lambda^{-1},
\end{gathered}
$$

for all $t \in I$. We note that the passage from (18) to (19) is obtained, thanks to (16) of Remark 2, with

$$
\left\|u_{f_{n+1}}(t)-u_{f_{n}}(t)\right\|=\left\|\int_{0}^{1} G(t, s)\left[f_{n+1}(s)-f_{n}(s)\right] d s\right\| \leq M_{G}\left\|f_{n+1}(t)-f_{n}(t)\right\|
$$

By $\left(\mathcal{H}_{2}\right)$, we have $d\left(f_{1}(t), F\left(t, u_{f_{1}}(t)\right)<l(t) \Lambda^{-1}, t \in I\right.$. Let us consider the multifunction $\Sigma_{1}: I \rightarrow c(E)$ defined by

$$
\Sigma_{1}(t)=\left\{v \in F\left(t, u_{f_{1}}(t)\right):\left\|v-f_{1}(t)\right\| \leq 2 l(t) \Lambda^{-1}\right\} .
$$

Clearly, $\Sigma_{1}$ is Lebesgue measurable with nonempty closed values. In view of the existence theorem of measurable selections (see [29]), there is a measurable function $f_{2}: I \rightarrow E$ such that $f_{2}(t) \in \Sigma_{1}(t)$ for all $t \in I$. This yields

$$
f_{2}(t) \in F\left(t, u_{f_{1}}(t)\right), \quad\left\|f_{2}(t)-f_{1}(t)\right\| \leq 2 l(t) \Lambda^{-1},
$$


for all $t \in I$. Thus, it is easy to see that $f_{2} \in L_{E}^{1}(I)$ and

$$
\left\|u_{f_{2}}(t)-u_{f_{1}}(t)\right\|=\left\|\int_{0}^{1} G(t, s)\left[f_{2}(s)-f_{1}(s)\right] d s\right\| \leq 2 \rho \Lambda^{-1},
$$

for all $t \in I$.

- Suppose that we have constructed integrable functions $f_{1}, f_{2}, \ldots, f_{n}$ such that

$$
\begin{gathered}
f_{i+1}(t) \in F\left(t, u_{f_{i}}(t)\right), t \in I, \\
\left\|f_{i+1}(t)-f_{i}(t)\right\| \leq(i+1) \rho^{i-1} l(t) \Lambda^{-1},
\end{gathered}
$$

for all $i=1,2, \ldots, n-1$. Then,

$$
\left\|u_{f_{i+1}}(t)-u_{f_{i}}(t)\right\|=\left\|\int_{0}^{1} G(t, s)\left[f_{i+1}(s)-f_{i}(s)\right] d s\right\| \leq(i+1) \rho^{i} \Lambda^{-1},
$$

for $i=1,2, \ldots, n-1$.

- The function $f_{n+1}$ is constructed as follows. We have

$$
\begin{aligned}
\left.d\left(f_{n}(t), F\left(t, u_{f_{n}}(t)\right)\right)\right) & \leq d_{H}\left(F\left(t, u_{f_{n-1}}(t)\right), F\left(t, u_{f_{n}}(t)\right)\right) \\
& \leq l(t)\left\|u_{f_{n}}(t)-u_{f_{n-1}}(t)\right\| \\
& \leq n \rho^{n-1} l(t) \Lambda^{-1} .
\end{aligned}
$$

The multifunction $\Sigma_{n}: I \rightarrow c(E)$, defined by

$$
\Sigma_{n}(t)=\left\{v \in F\left(t, u_{n}(t)\right):\left\|v-f_{n}(t)\right\| \leq(n+1) \rho^{n-1} l(t) \varepsilon \Lambda^{-1}\right\},
$$

is Lebesgue measurable with nonempty closed values. Thus, there exists a measurable function $f_{n+1}$ such that

$$
f_{n+1}(t) \in F\left(t, u_{f_{n}}(t)\right), \quad\left\|f_{n+1}(t)-f_{n}(t)\right\| \leq(n+1) \rho^{n-1} l(t) \Lambda^{-1},
$$

for all $t \in I$. Then, it is clear that, for all $t \in I$,

$$
\left\|u_{f_{n+1}}(t)-u_{f_{n}}(t)\right\|=\left\|\int_{0}^{1} G(t, s)\left[f_{n+1}(s)-f_{n}(s)\right] d s\right\| \leq(n+1) \rho^{n} \Lambda^{-1},
$$

Thus, such a sequence $\left\{f_{n}\right\}_{n=1}^{\infty}$ with the required properties exists.

Step 2. It follows that, for all $n \geq 1$, we have

$$
\left\|f_{n+1}-f_{n}\right\|_{L_{E}^{1}(I)}=\int_{0}^{1}\left\|f_{n+1}(t)-f_{n}(t)\right\| d t \leq(n+1) \rho^{n-1}\|l\|_{L_{\mathbf{R}^{+}}^{1}(I)} \Lambda^{-1} .
$$


On the other hand, by $\rho<1$ the series $\sum_{n=1}^{\infty}(n+1) \rho^{n-1}$ is convergent (using d'Alembert's ratio test). Now, we assert that $\left\{f_{n}(\cdot)\right\}_{n=1}^{\infty}$ is a Cauchy sequence in $L_{E}^{1}(I)$. Indeed, using (10), for $n, m \in \mathbf{N}$ such that $m>n$, we have the estimate

$$
\begin{aligned}
\left\|f_{m}-f_{n}\right\|_{L_{E}^{1}(I)} & \leq\left\|f_{n+1}-f_{n}\right\|_{L_{E}^{1}(I)}+\left\|f_{n+2}-f_{n+1}\right\|_{L_{E}^{1}(I)}+\cdots+\left\|f_{m}-f_{m-1}\right\|_{L_{E}^{1}(I)} \\
& \leq\left[(n+1) \rho^{n-1}+(n+2) \rho^{n}+\cdots+m \rho^{m-2}\right]\|l\|_{L_{\mathbf{R}^{+}}^{1}(I)} \Lambda^{-1} \\
& \leq\left(\sum_{k=n}^{\infty}(k+1) \rho^{k-1}\right)\|l\|_{L_{\mathbf{R}^{+}}^{1}(I)} \Lambda^{-1}
\end{aligned}
$$

Letting $n \rightarrow \infty$ in the above inequality, we see that $\left\|f_{m}-f_{n}\right\|_{L_{E}^{1}(I)}$ goes to 0 when $m, n$ goes to $\infty$. Since the normed space $L_{E}^{1}(I)$ is complete, $\left(f_{n}\right)$ norm converges to an element $f \in L_{E}^{1}(I)$. By the properties of our Green function and the definition of $u_{f_{n}}$, we conclude that $u_{f_{n}}$ pointwise converge with respect to the norm topology to $u_{f}$

$$
u_{f}(t)=\int_{0}^{1} G(t, s) f(s) d s, \forall t \in I .
$$

Now, we claim that $f(t) \in F\left(t, u_{f}(t)\right)$, a.e. $t \in I$. Let us write

$$
\begin{aligned}
d\left(f(t), F\left(t, u_{f}(t)\right) \leq\right. & \mid d\left(f(t), F\left(t, u_{f}(t)\right)\right)-d\left(f_{n}(t), F\left(t, u_{f}(t)\right) \mid\right. \\
& +d\left(f_{n}(t), F\left(t, u_{f}(t)\right) .\right.
\end{aligned}
$$

On the other hand,

$$
\mid d\left(f(t), F\left(t, u_{f}(t)\right)-d\left(f_{n}(t), F\left(t, u_{f}(t)\right)\right) \mid \leq\left\|f(t)-f_{n}(t)\right\|,\right.
$$

and, by $f_{n}(t) \in F\left(t, u_{f_{n-1}}(t)\right), t \in I$, we have

$$
\begin{aligned}
d\left(f_{n}(t), F\left(t, u_{f}(t)\right)\right. & \leq d_{H}\left(F\left(t, u_{f_{n-1}}(t)\right), F\left(t, u_{f}(t)\right)\right) \\
& \leq l(t)\left\|u_{f_{n-1}}(t)-u_{f}(t)\right\| .
\end{aligned}
$$

Since $\left(f_{n}\right)_{n \in \mathbf{N}}$ norm converges to $f \in L_{E}^{1}(I)$, we may, by extracting subsequences, assume that $\left\|f_{n}(t)-f(t)\right\|_{E} \rightarrow 0$ a.e. Now, passing to the limit when $n \rightarrow \infty$ in the preceding inequality, we get

$$
d\left(f(t), F\left(t, u_{f}(t)\right)\right)=0 \quad \text { a.e. } t \in I
$$

This implies that $f(t) \in F\left(t, u_{f}(t)\right)$, a.e.t $\in I$ because $F$ is closed valued. Thus, by Lemma 9, we have shown that $u_{f}$ is a solution of the problem

$$
\left\{\begin{array}{l}
D^{\alpha} u_{f}(t)+\lambda D^{\alpha-1} u_{f}(t) \in F\left(t, u_{f}(t)\right), \text { a.e. } t \in I \\
\left.I_{0^{+}}^{\beta} u_{f}(t)\right|_{t=0}=0, \quad u_{f}(1)=I_{0^{+}}^{\gamma} u_{f}(1)
\end{array}\right.
$$

The proof of theorem is complete.

A relaxation theorem is available using the machinery developed in [36] Theorem 4.2 and Lemma 9.

Theorem 10. Relaxation Assume that $E$ is a separable Banach space. Let $F: I \times E \rightarrow E$ be a closed valued $\mathcal{L}(I) \otimes \mathcal{B}(E)$-measurable mapping such that 
$\left(\mathcal{H}_{1}\right): d_{H}(F(t, x), F(t, y)) \leq l(t)\|x-y\|$ for all $t, x, y$ where $\left.l \in L_{\mathbf{R}}^{1}(I)\right)$ such that $\rho:=M_{G}\|l\|_{L_{\mathbf{R}}^{1}(I)}<1$. Assume further that

$\left(\mathcal{H}_{2}\right)$ : there exists $g \in L_{E}^{1}(I)$ such that $d\left(g(t), F\left(t, u_{g}(t)\right)\right)<\frac{l(t)}{\sum_{n=1}^{\infty} n \rho^{n-1}}$ where $u_{g}(t)=$ $\int_{0}^{1} G(t, s) g(s) d s, \forall t \in I$.

$\left(\mathcal{H}_{3}\right): d(0, F(t, x))<c(t)(1+\|x\|), \forall(t, x) \in I \times E$ where $c$ is a positive integrable function.

Then, the following holds:

(a)

$$
\left(\mathcal{P}_{F}\right)\left\{\begin{array}{l}
D^{\alpha} u(t)+\lambda D^{\alpha-1} u(t) \in F(t, u(t)) \text {, a.e. } t \in I \\
\left.I_{0^{+}}^{\beta} u(t)\right|_{t=0}=0, \quad u(1)=I_{0^{+}}^{\gamma} u(1)
\end{array}\right.
$$

and

$$
\left(\mathcal{P}_{\overline{c o} F}\right)\left\{\begin{array}{l}
D^{\alpha} u(t)+\lambda D^{\alpha-1} u(t) \in \overline{c o} F(t, u(t)), \text { a.e. } t \in I \\
\left.I_{0^{+}}^{\beta} u(t)\right|_{t=0}=0, \quad u(1)=I_{0^{+}}^{\gamma} u(1)
\end{array}\right.
$$

have at least a solution in $W_{B, E}^{\alpha, 1}(I)$.

(b) Let $\left.f_{0} \in L_{E}^{1}(I)\right)$ such that

$$
\begin{gathered}
f_{0}(t) \in \overline{c o} F\left(t, u_{f_{0}}(t)\right) \\
u_{f_{0}}(t)=\int_{0}^{1} G(t, s) f_{0}(s) d s, \forall t \in I
\end{gathered}
$$

Then, for every $\varepsilon>0$, there exists $f \in L_{E}^{1}(I)$ such that

$$
\begin{gathered}
f(t) \in F\left(t, u_{f}(t)\right), \quad \text { a.e. } \\
u_{f}(t)=\int_{0}^{1} G(t, s) f(s) d s, \forall t \in I
\end{gathered}
$$

and

$$
\sup _{t \in I}\left\|u_{f}(t)-u_{f_{0}}(t)\right\| \leq \varepsilon
$$

Proof. We will proceed in several steps.

Step 1. (a) follows from Theorem 9 applied to both $F$ and $\overline{c o} F$ taking account of $\left(\mathcal{H}_{1}\right)-\left(\mathcal{H}_{2}\right)$. Let $u_{f_{0}}(\cdot)$ be a $W_{B, E}^{\alpha, 1}(I)$-solution of the problem $\left(\mathcal{P}_{\overline{c o} F}\right)$ that is, $u_{f_{0}} \in \mathcal{S}_{\mathcal{P}_{\overline{\bar{c}} F}}$

$$
\begin{gathered}
f_{0}(t)=\overline{c o} F\left(t, u_{f_{0}}(t)\right), \text { a.e. } t \in I, \\
u_{f_{0}}(t):=\int_{0}^{1} G(t, s) f_{0}(s) d s, \forall t \in I
\end{gathered}
$$

Let $S_{F}^{1}$ and $S_{\overline{c o} F}^{1}$ denote the set of all $L_{E}^{1}(I)$-selections of the set valued mappings $t \rightarrow F\left(t, u_{f_{0}}(t)\right)$ and $t \rightarrow \overline{c o} F\left(t, u_{f_{0}}(t)\right)$ By $\left(\mathcal{H}_{3}\right)$, the multifunction $t \mapsto F\left(t, u_{f_{0}}(t)\right)$ is closed valued and integrable:

$$
d\left(0, F\left(t, u_{f_{0}}(t)\right)<c(t)\left(1+\left\|u_{f_{0}}(t)\right\|\right)\right.
$$

so that $S_{F}^{1}$ is non empty. Then, according to Hiai-Umegaki [37], $S_{\overline{c o} F}^{1}=\overline{c o} S_{F}^{1}$ where $\overline{c o}$ is taken in $L_{E}^{1}(I)$. This equality along with $f_{0}(t) \in \overline{c o} F\left(t, u_{f_{0}}(t)\right)$, a.e. $t \in I$ yields $f_{0} \in \overline{c o} S_{F}^{1}$. Let $\varepsilon>0$. There exists $g_{\varepsilon} \in L_{E}^{1}(I)$ such that $g_{\varepsilon} \in \operatorname{coS}_{F}^{1}$ and $\left\|f_{0}-g_{\varepsilon}\right\|_{L_{E}^{1}(I)} \leq \frac{1}{2} \varepsilon \Lambda^{-1} M_{G}^{-1}$ so that

$$
\left\|u_{f_{0}}(t)-u_{g_{\varepsilon}}(t)\right\|<\frac{1}{2} \varepsilon \Lambda^{-1}
$$


As $g_{\varepsilon} \in \operatorname{coS}_{F}^{1}$, then $g_{\varepsilon}=\sum_{i=1}^{n} \lambda_{i} f_{I}$ with $f_{i} \in L_{E}^{1}(I), f_{i}(t) \in F\left(t, u_{f_{0}}(t)\right), \lambda_{i} \geq 0, \sum_{i=1}^{n} \lambda_{i}=1$. Let $\Phi(t):=\left\{f_{i}(t: 1 \leq i \leq n\}\right.$, then $\Phi(t)$ is a compact valued integrably bounded mapping with $|\Phi(t)| \leq r(t):=\sup _{1 \leq i \leq n}\left|f_{i}(t)\right|$. Then, from [38], there exists

$$
\left.h_{1} \in L_{E}^{1}(I), h_{1}(t) \in \Phi(t) \subset F\left(t, u_{f_{0}}(t)\right)\right), \forall t \in I
$$

such that

$$
\sup _{0 \leq t<\tau \leq 1}\left\|\int_{t}^{\tau}\left[h_{1}(s)-g_{\varepsilon}(s)\right] d s\right\| \leq \frac{1}{2} \varepsilon M_{G}^{-1} \Delta^{-1}
$$

so that

$$
\left\|u_{h_{1}}(t)-u_{g_{\varepsilon}}(t)\right\|=\| \int_{0}^{1} G(t, s)\left[h_{1}(s)-g_{\varepsilon}(s) d s\left\|\leq M_{G}\right\| \int_{0}^{1}\left[h_{1}(s)-g_{\varepsilon}(s) d s \| \leq \frac{1}{2} \varepsilon \Delta^{-1} .\right.\right.
$$

Consequently,

$$
\left\|u_{h_{1}}(t)-u_{f_{0}}(t)\right\| \leq \varepsilon \Delta^{-1}
$$

Step 2. We shall construct inductively sequence $\left\{h_{n}(\cdot)\right\}_{n=1}^{\infty}$ such that the following conditions are fulfilled, for all $n \geq 1$,

$$
\begin{gathered}
h_{n} \in L_{E}^{1}(I) \text { and } h_{n+1}(t) \in F\left(t, u_{h_{n}}(t)\right), t \in I, \\
\left\|h_{n+1}(t)-h_{n}(t)\right\| \leq(n+1) \rho^{n-1} l(t) \varepsilon \Lambda^{-1}, \\
\left\|u_{h_{n+1}}(t)-u_{h_{n}}(t)\right\|=\left\|\int_{0}^{1} G(t, s)\left[h_{n+1}(s)-h_{n}(s)\right] d s\right\| \leq(n+1) \rho^{n} \varepsilon \Lambda^{-1}, t \in I
\end{gathered}
$$

- The multifunction $F\left(\cdot, u_{h_{1}}(\cdot)\right)$ is Lebesgue-measurable and

$$
d_{H}\left(F\left(t, u_{h_{1}}(t)\right), F\left(t, u_{f_{0}}(t)\right)\right) \leq l(t)\left\|u_{h_{1}}(t)-u_{f_{0}}(t)\right\|
$$

This implies that, for $t \in I$,

$$
d_{H}\left(F\left(t, u_{h_{1}}(t)\right), F\left(t, u_{f_{0}}(t)\right)\right) \leq l(t) \varepsilon \Lambda^{-1},
$$

As $h_{1}(t) \in F\left(t, u_{f_{0}}(t)\right)$, we have $d\left(h_{1}(t), F\left(t, u_{h_{1}}(t)\right) \leq l(t) \varepsilon \Lambda^{-1}, t \in I\right.$. Let us consider the multifunction $\Sigma_{1}: I \rightarrow c(E)$ defined by

$$
\Sigma_{1}(t)=\left\{v \in F\left(t, u_{h_{1}}(t)\right):\left\|v-h_{1}(t)\right\| \leq 2 l(t) \varepsilon \Lambda^{-1}\right\} .
$$

Clearly, $\Sigma_{1}$ is Lebesgue measurable with nonempty closed values. In view of the existence theorem of measurable selections (see [29]), there is a measurable function $h_{2}: I \rightarrow E$ such that $h_{2}(t) \in \Sigma_{1}(t)$ for all $t \in I$. This yields

$$
h_{2}(t) \in F\left(t, u_{h_{1}}(t)\right), \quad\left\|h_{2}(t)-h_{1}(t)\right\| \leq 2 l(t) \varepsilon \Lambda^{-1},
$$

for all $t \in I$. Thus, it is easy to see that $h_{2} \in L_{E}^{1}(I)$ and

$$
\left\|u_{h_{2}}(t)-u_{h_{1}}(t)\right\|=\left\|\int_{0}^{1} G(t, s)\left[h_{2}(s)-h_{1}(s)\right] d s\right\| \leq 2 \rho \varepsilon \Lambda^{-1},
$$

for all $t \in I$.

- Suppose that we have constructed integrable functions $h_{1}, h_{2}, \ldots, h_{n}$ such that

$$
h_{i+1}(t) \in F\left(t, u_{h_{i}}(t)\right) \text {, a.e.t } \in I \text {, }
$$




$$
\left\|h_{i+1}(t)-h_{i}(t)\right\| \leq(i+1) \rho^{i-1} l(t) \varepsilon \Lambda^{-1},
$$

for all $i=1,2, \ldots, n-1$. Then,

$$
\left\|u_{h_{i+1}}(t)-u_{h_{i}}(t)\right\|=\left\|\int_{0}^{1} G(t, s)\left[h_{i+1}(s)-h_{i}(s)\right] d s\right\| \leq(i+1) \rho^{i} \varepsilon \Lambda^{-1},
$$

for $i=1,2, \ldots, n-1$.

- The function $h_{n+1}$ is constructed as follows. We have

$$
\begin{gathered}
d\left(h_{n}(t), F\left(t, u_{h_{n}}(t)\right)\right) \leq d_{H}\left(F\left(t, u_{h_{n-1}}(t)\right), F\left(t, u_{h_{n}}(t)\right)\right) \\
\leq l(t)\left\|u_{h_{n}}(t)-u_{h_{n-1}}(t)\right\| \leq n \rho^{n-1} l(t) \varepsilon \Lambda^{-1}
\end{gathered}
$$

The multifunction $\Sigma_{n}: I \rightarrow c(E)$, defined by

$$
\Sigma_{n}(t)=\left\{v \in F\left(t, u_{h_{n}}(t)\right):\left\|v-h_{n}(t)\right\| \leq(n+1) \rho^{n-1} l(t) \varepsilon \Lambda^{-1}\right\},
$$

is Lebesgue measurable with nonempty closed values. Thus, there exists a measurable function $h_{n+1}$ such that

$$
h_{n+1}(t) \in F\left(t, u_{h_{n}}(t)\right), \quad\left\|h_{n+1}(t)-h_{n}(t)\right\| \leq(n+1) \rho^{n-1} l(t) \varepsilon \Lambda^{-1},
$$

for all $t \in I$. Then, it is clear that, for all $t \in I$,

$$
\left\|u_{h_{n+1}}(t)-u_{h_{n}}(t)\right\|=\left\|\int_{0}^{1} G(t, s)\left[h_{n+1}(s)-h_{n}(s)\right] d s\right\| \leq(n+1) \rho^{n} \varepsilon \Lambda^{-1},
$$

Thus, a sequence $\left\{h_{n}\right\}_{n=1}^{\infty}$ satisfying (27)-(29)exists.

Step 3. It follows from (28) that, for all $n \geq 1$, we have

$$
\left\|h_{n+1}-h_{n}\right\|_{L_{E}^{1}(I)}=\int_{0}^{1}\left\|h_{n+1}(t)-h_{n}(t)\right\| d t \leq(n+1) \rho^{n-1}\|l\|_{L_{\mathbf{R}^{+}}^{1}(I)} \varepsilon \Lambda^{-1} .
$$

On the other hand, by $\rho<1$, the series $\sum_{n=1}^{\infty}(n+1) \rho^{n-1}$ is convergent (using d'Alembert's ratio test). Now, we assert that $\left\{h_{n}(\cdot)\right\}_{n=1}^{\infty}$ is a Cauchy sequence in $L_{E}^{1}(I)$. Indeed, using (30), for $n, m \in \mathbf{N}$, such that $m>n$, we have the estimate

$$
\begin{aligned}
\left\|h_{m}-h_{n}\right\|_{L_{E}^{1}(I)} & \leq\left\|h_{n+1}-h_{n}\right\|_{L_{E}^{1}(I)}+\left\|h_{n+2}-h_{n+1}\right\|_{L_{E}^{1}(I)}+\cdots+\left\|h_{m}-h_{m-1}\right\|_{L_{E}^{1}(I)} \\
& \leq\left[(n+1) \rho^{n-1}+(n+2) \rho^{n}+\cdots+m \rho^{m-2}\right]\|1\|_{L_{\mathbf{R}^{+}}^{1}(I)} \varepsilon \Lambda^{-1} \\
& \leq\left(\sum_{k=n}^{\infty}(k+1) \rho^{k-1}\right)\|l\|_{L_{\mathbf{R}^{+}}^{1}(I)} \varepsilon \Lambda^{-1}
\end{aligned}
$$

Letting $n \rightarrow \infty$ in the above inequality, we see that $\left\|h_{m}-h_{n}\right\|_{L_{E}^{1}(I)}$ goes to 0 when $m, n$ goes to $\infty$. Since the normed space $L_{E}^{1}(I)$ is complete, $\left(h_{n}\right)$ norm converges to an element $f \in L_{E}^{1}(I)$. By the properties of our Green function and the definition of $u_{h_{n}}$, we conclude that $u_{h_{n}}$ pointwise converges with respect to the norm topology to $u_{f}$ where

$$
u_{f}(t)=\int_{0}^{1} G(t, s) f(s) d s
$$


Moreover, from (29), we deduce that

$$
\begin{gathered}
\left\|u_{h_{n}}(t)-u_{f_{0}}(t)\right\| \leq\left\|u_{h_{1}}(t)-u_{f_{0}}(t)\right\|+\left\|u_{h_{2}}(t)-u_{h_{1}}(t)\right\|+\ldots+\left\|u_{h_{n}}(t)-u_{h_{n-1}}(t)\right\| \\
\leq\left(\sum_{j=1}^{n} j \rho^{j-1}\right) \varepsilon \Lambda^{-1}
\end{gathered}
$$

for all $t \in I$. Recall that $\Lambda=\sum_{n=1}^{\infty} n \rho^{n-1}$. Thus, by letting $n \rightarrow \infty$ in the last inequality, we get

$$
\left\|u_{f}-u_{f_{0}}\right\|_{C_{E}(I)}=\max _{t \in I}\left\|u_{f}(t)-u_{f_{0}}(t)\right\| \leq \varepsilon .
$$

Now, we claim that $f(t) \in F\left(t, u_{f}(t)\right)$, a.e. $t \in I$. Let us write

$$
\begin{aligned}
d\left(f(t), F\left(t, u_{f}(t)\right) \leq\right. & \mid d\left(f(t), F\left(t, u_{f}(t)\right)-d\left(h_{n}(t), F\left(t, u_{f}(t)\right) \mid\right.\right. \\
& +d\left(h_{n}(t), F\left(t, u_{f}(t)\right) .\right.
\end{aligned}
$$

On the other hand,

$$
\mid d\left(f(t), F\left(t, u_{f}(t)\right)-d\left(h_{n}(t), F\left(t, u_{f}(t)\right)\right) \mid \leq\left\|f(t)-h_{n}(t)\right\|,\right.
$$

and, by $h_{n}(t) \in F\left(t, u_{h_{n-1}}(t)\right), t \in I$, we have

$$
\begin{aligned}
d\left(h_{n}(t), F\left(t, u_{f}(t)\right)\right. & \leq d_{H}\left(F\left(t, u_{h_{n-1}}(t)\right), F\left(t, u_{f}(t)\right)\right) \\
& \leq l(t)\left\|u_{h_{n-1}}(t)-u_{f}(t)\right\|
\end{aligned}
$$

Since $\left(h_{n}\right)_{n \in \mathbf{N}}$ norm converges to $f \in L_{E}^{1}(I)$ we may, by extracting subsequences, assume that $\left\|h_{n}(t)-f(t)\right\|_{E} \rightarrow 0$ a.e. Now, passing to the limit when $n \rightarrow \infty$ in (31)-(33), we get

$$
d\left(f(t), F\left(t, u_{f}(t)\right)=0 \text { a.e. } t \in I\right.
$$

This implies that $f(t) \in F\left(t, u_{f}(t)\right)$, a.e. $t \in I$ because $F$ is closed valued. Hence, $u_{f}$ is a solution of the problem $\left(\mathcal{P}_{F}\right)$, satisfying the required density property. The proof of theorem is complete.

\section{Conclusions}

In the context of separable Hilbert space, our algorithm and tools are fairly general and they allow for treating several variants of system of fractional differential inclusion coupled with a time and state dependent maximal monotone operators with Lipschitz perturbation, in particular the second order solution of evolution inclusion governed time and state dependent maximal monotone operators with Lipschitz perturbation. Our results contain novelties. Nevertheless, there are several issues-for instance, the existence of solutions for the case of closed unbounded Lipschitz perturbation that is needed in the optimal control.

Author Contributions: All authors have contributed equally to this work for writing, review and editing. All authors have read and agreed to the published version of the manuscript.

Funding: This research received no external funding.

Conflicts of Interest: The authors declare no conflict of interest. 


\section{References}

1. Kilbas, A.A.; Srivastava, H.M.; Trujillo, J.J. Theory and Applications of Fractional Differential Equations; Math. Studies 204; Elsevier: Amsterdam, The Netherlands, 2006.

2. Miller, K.S.; Ross, B. An Introduction to the Fractional Calculus and Fractional Differential Equations; Wiley: New York, NY, USA, 1993.

3. Podlubny, I. Fractional Differential Equations; Academic Press: New York, NY, USA, 1999.

4. Samko, S.G.; Kilbas, A.A.; Marichev, O.I. Fractional Integrals and Derivatives: Theory and Applications; Gordon and Breach: New York, NY, USA, 1993.

5. Vrabie, I.L. Compactness Methods for Nonlinear Evolution Equations, Pitman Monographs and Surveys in Pure and Applied Mathematics (Vol. 32), Longman Scientific and Technical; John Wiley: New York, NY, USA, 1987.

6. Graef, J.R.; Henderson, J.; Ouahab, A. Impulsive Differential Inclusions. A Fixed Point Approach; De Gruyter Series in Nonlinear Analysis and Applications 20; de Gruyter: Berlin, Germany, 2013.

7. Zhou, Y. Basic Theory of Fractional Differential Equations; World Scientific Publishing: Singapore, 2014.

8. Ahmad, B.; Nieto, J. Riemann-Liouville fractional differential equations with fractional boundary conditions. Fixed Point Theory 2012, 13, 329-336.

9. Ouahab, A. Some results for fractional boundary value problem of differential inclusions. Nonlinear Anal. 2008, 69, 3877-3896.

10. Phung, P.H.; Truong, L.X. On a fractional differential inclusion with integral boundary conditions in Banach space. Fract. Calc. Appl. Anal. 2013, 16, 538-558.

11. El-Sayed, A.M.A.; Ibrahim, A.G. Set-valued integral equations of arbitrary (fractional) order. Appl. Math. Comput. 2001, 118, 113-121.

12. Castaing, C.; Truong, L.X.; Phung, P.D. On a fractional differential inclusion with integral boundary condition in Banach spaces. J. Nonlinear Convex Anal. 2016, 17, 441-471.

13. Benchohra, M.; Graef, J.; Mostefai, F.Z. Weak solutions for boundary-value problems with nonlinear fractional differential inclusions. Nonlinear Dyn. Syst. Theory 2011, 11, 227-237.

14. El-Sayed, A.M.A. Nonlinear functional differential equations of arbitrary orders. Nonlinear Anal. 1998, 33, 181-186.

15. Cernea, A. On a fractional differential inclusion with boundary condition. Stud. Univ. Babes-Bolyai Math 2010, LV, 105-113.

16. Agarwal, R.P.; Arshad, S.; O’Reagan, D.; Lupulescu, V. Fuzzy Fractional Integral Equations under Compactness type condition. Frac. Calc. Appl. Anal. 2012, 15, 572-590.

17. Benchohra, M.; Henderson, J.; Ntouyas, S.K.; Ouahab, A. Existence results for fractional functional differential inclusions with infinite delay and applications to control theory. Fract. Calc. Appl. Anal. 2008, 11, 35-56.

18. Castaing, C.; Monteiro Marques, M.D.P.; Saidi, S. Evolution problems with time-dependent subdifferential operators. Adv. Math. Econ. 2020, 23, 1-39.

19. Barbu, V. Nonlinear Semigroups and Differential Equations in Banach Spaces; Noordhoff International Publishing: Leyden, The Netherlands, 1976.

20. Brezis, H. Opérateurs Maximaux Monotones; Elsevier: Amsterdam, The Netherlands, 1973.

21. Vladimirov, A.A. Nonstationnary dissipative evolution equation in Hilbert space. Nonlinear Anal. 1991, 17, 499-518.

22. Azzam-Laouir, D.; Belhoula, W.; Castaing, C.; Monteiro Marques, M.D.P. Multi-valued perturbation to evolution problems involving time dependent maximal monotone operators. Evol. Equ. Control Theory 2020, 9, 219-254.

23. Kunze, M.; Monteiro Marques, M.D.P. BV solutions to evolution problems with time-dependent domains. Set-Valued Anal. 1997, 5, 57-72.

24. Castaing, C.; Ibrahim A.G.; Yarou, M. Some contributions to nonconvex sweeping process. J. Nonlinear Convex Anal. 2009, 10, 1-20.

25. Idzik, A. Almost fixed points theorems. Proc. Am. Math. Soc. 1988, 104, 779-784.

26. Park, S. Fixed points of approximable or Kakutani maps. J. Nonlinear Convex Anal. 2006 , 7, 1-17.

27. Castaing, C. Quelques résultats de compacité liés a l'intégration. CR Acad. Sci. Paris 1970, 270, 1732-1735; reprinted in Bull. Soc. Math. France 1972, 31, 73-81. 
28. Castaing, C.; Godet-Thobie, C.; Phung, P.D.; Truong, L.X. On fractional differential inclusions with nonlocal boundary conditions. Fract. Calc. Appl. Anal. 2019, 22, 444-478.

29. Castaing, C.; Valadier, M. Convex Analysis and Measurable Multifunctions; Lecture Notes in Mathematics, 580; Springer: Berlin/Heidelberg, Germany, 1977.

30. Monteiro Marques, M.D.P. Differential Inclusions Nonsmooth Mechanical Problems, Shocks and Dry Friction. In Progress in Nonlinear Differential Equations and Their Applications; Springer: Berlin, Germany, 1993; Volume 9.

31. Moreau, J.J. Evolution problem asssociated with a moving convex set in a Hilbert space. J. Differ. Equ. 1977, 26, 347-374.

32. Adly, S.; Haddad, T.; Thibault, L. Convex sweeping process in the framework of measure differential inclusions and evolution variational inequalities. Math. Program 2014, 148, 5-47.

33. Castaing, C. Weak compactness and convergences in Bochner and Pettis integration. Vietnam J. Math. 1996, 24, 241-286.

34. Castaing, C.; Marques, M.D.P.; Raynaud de Fitte, P. Second order evolution problems with time dependent maximal monotone operator. Adv. Math. Econ. 2018, 23, 25-77

35. Kenmochi, N. Solvability of nonlinear evolution equations with time-dependent constraints and applications. Bull. Fac. Educ. Chiba Univ. 1981, 30, 1.

36. Castaing, C.; Truong, L.X. Some Topological Properties of Solution Sets in a Second Order Differential Inclusion with m-point Boundary Conditions. Set-Valued Var. Anal. 2012, 20, 249-277.

37. Hiai, F.; Umegaki, H. Integrals, conditional expectations, and martingales of multivalued functions. J. Multivar. Anal. 1977, 7, 149-182.

38. Tolstonogov, A.A.; Tolstonogov, D. Lp Continuous Extreme Selectors of Multifunctions with Decomposable Values: Relaxation Theorems. Set-Valued Anal. 1996, 4, 237-269.

(C) 2020 by the authors. Licensee MDPI, Basel, Switzerland. This article is an open access article distributed under the terms and conditions of the Creative Commons Attribution (CC BY) license (http://creativecommons.org/licenses/by/4.0/). 